\title{
Mixing in Circular and Non-circular Jets in Crossflow
}

\author{
Salewski, Mirko; Stankovic, D.; Fuchs, L.
}

\section{Published in:}

Flow, Turbulence and Combustion

Link to article, DOI:

10.1007/s10494-007-9119-x

Publication date:

2008

Link back to DTU Orbit

Citation (APA):

Salewski, M., Stankovic, D., \& Fuchs, L. (2008). Mixing in Circular and Non-circular Jets in Crossflow. Flow, Turbulence and Combustion, 80, 255-283. https://doi.org/10.1007/s10494-007-9119-x

\section{General rights}

Copyright and moral rights for the publications made accessible in the public portal are retained by the authors and/or other copyright owners and it is a condition of accessing publications that users recognise and abide by the legal requirements associated with these rights.

- Users may download and print one copy of any publication from the public portal for the purpose of private study or research.

- You may not further distribute the material or use it for any profit-making activity or commercial gain

- You may freely distribute the URL identifying the publication in the public portal

If you believe that this document breaches copyright please contact us providing details, and we will remove access to the work immediately and investigate your claim. 
submitted to

Flow, Turbulence, and Combustion

\title{
Mixing in Circular and Non-Circular Jets in Crossflow
}

\author{
M. Salewski, D. Stankovic and L. Fuchs \\ Div. Fluid Mechanics, Lund University, SE-221 00 Lund, Sweden \\ September 2007
}

\begin{abstract}
Coherent structures and mixing in the flow field of a jet in crossflow have been studied using computational (Large Eddy Simulation) and experimental (Particle Image Velocimetry and Laser-Induced Fluorescence) techniques. The mean scalar fields and turbulence statistics as determined by both are compared for circular, elliptic, and square nozzles. For the latter configurations, effects of orientation are considered. The computations reveal that the distribution of a passive scalar in a cross-sectional plane can be singleor double-peaked, depending on the nozzle shape and orientation. A proper orthogonal decomposition of the transverse velocity indicates that coherent structures may be responsible for this phenomenon. Nozzles which have a single-peaked distribution have stronger modes in transverse direction. The global mixing performance is superior for these nozzle types. This is the case for the blunt square nozzle and for the elliptic nozzle with high aspect ratio. It is further demonstrated that the flow field contains large regions in which a passive scalar is transported up the mean gradient (counter-gradient transport) which implies failure of the gradient diffusion hypothesis.
\end{abstract}

\section{Introduction}

Transverse jets, or jets in crossflow (JICF), are generic flows which are often employed in technical applications where fast mixing is desired, for example fuel injection into burners for gas turbines (GT) operating in the premixed combustion mode. The need to have high thermodynamic efficiency and low pollutant emissions poses a design challenge. High thermodynamic efficiency is necessary to maintain low fuel consumption rates and low absolute amounts of emissions per unit power output. This strive for high thermodynamic efficiency implies increasing levels of inlet pressures and temperatures, favoring pollutant formation, most importantly $N O_{x}$. Current GT burners with premixed flames utilize fuel injection into the main stream. The mixing efficiency determines the size of the mixing zone and thereby affects the dimensions of the combustor. For airborne GT combustors, the size is a significant design parameter.

The flow field of JICF is highly unsteady and has several typical and distinguishable structures. Curved mixing layers form between the jet and the crossflow. The term "crossflow" is used here for the main channel flow, in which the tiny jet is injected, following the common terminology. The mixing layers are subject to Kelvin-Helmholtz instability on the windward and leeward sides of the jet, leading to formation of unsteady shear layer vortices. On the lateral sides of the jet, however, the mixing layer is skewed and the instability is therefore of different character [1]. Instead of regular roll-up of vortices as for example can be observed in free jets [2], a pair of counter-rotating vortices, which has 


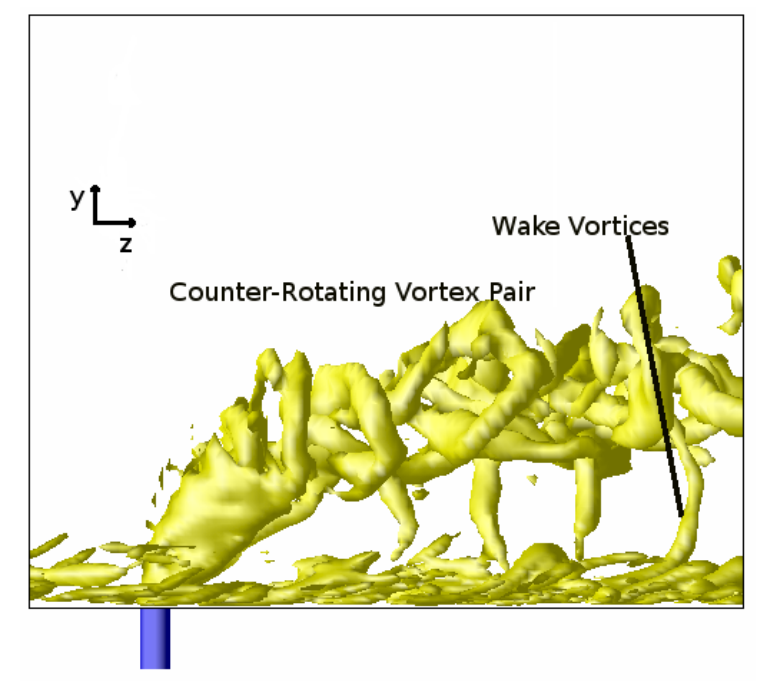

Figure 1: $\lambda_{2}$ vortex visualization [8] of the counter-rotating vortex pair and wake vortices

been dubbed "quasi-steady hanging vortices" , emanates from the lateral nozzle edges [1]. Due to these vortices, crossflow fluid is engulfed into the jet. This near-field entrainment mechanism contributes to the deflection of the jet in the near-field [3] and to the excellent mixing properties of the JICF as compared to a free jet. After few nozzle diameters the jet splits into two branches [4], the lower of which evolves into the eminent counterrotating vortex pair (CVP), the dominant feature in the far-field. The CVP meanders in the turbulent flow, and it can be detected up to 1000 nozzle diameters downstream of the jet injection [5]. On the windward side of the jet, horseshoe vortices form due to lateral stretching of boundary layer vorticity, analog to horseshoe vortices ahead of a solid cylinder [6]. In the wake, upright vortices (sometimes referred to as "wake vortices") form intermittently between the CVP and the boundary layer [7]. Close to the wall, there is a pair of secondary vortices, rotating in the opposite direction of the CVP. The large coherent structures dominate the large-scale mixing and mass-transport. Small-scale mixing, on the other hand, is effected by turbulence. Comprehensive knowledge about the coherent structures and their consequences with respect to mixing is therefore required. Figure 1 is a side view snapshot of the coherent vortices in the flow field, visualized with the $\lambda_{2}$-criterion [8]. The figure illustrates the CVP, the upright tornado-like wake vortices, and the vortices close to the wall. It is apparent that the CVP undulates in the turbulent flow and interacts with the intermittent wake vortices which in turn interact with the boundary layer and the vortices therein $[1,7,9,10]$. Figure 2 shows the streamwise vorticity of the JICF. The dark region is a positive vorticity isosurface and the light region a negative isosurface, clearly revealing the CVP. One notes that the streamwise vorticity is convected downstream forming the two vortices of the CVP.

A leading parameter determining the jet lift-off is the velocity ratio $R$ (Eq. 1) or, if the densities in the jet and the crossflow are different, the effective velocity ratio $R_{e}$, which is defined as the square root of the momentum flux ratio (Eq. 2).

$$
\begin{aligned}
R & =\frac{V_{\text {jet }}}{V_{\text {crossflow }}} \\
R_{e} & =\sqrt{\frac{\left(\rho V^{2}\right)_{\text {jet }}}{\left(\rho V^{2}\right)_{\text {crossflow }}}}
\end{aligned}
$$

Muppidi and Mahesh [11] demonstrate by direct numerical simulations (DNS) that jets penetrate deeper into the crossflow if the crossflow boundary layer and the jet boundary 


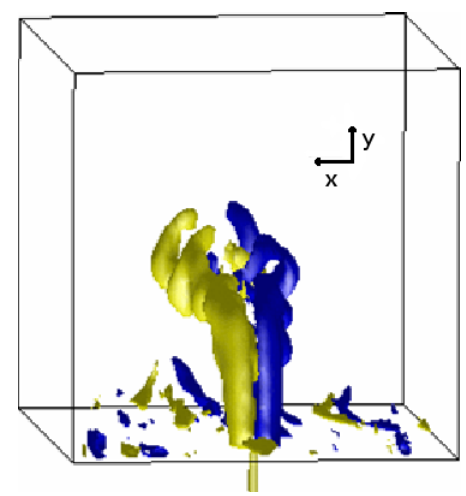

Figure 2: Counter-Rotating vortex pair shown by positive (dark) and negative (light) streamwise vorticity isosurfaces

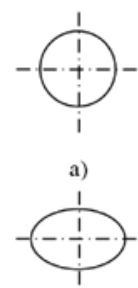

b)

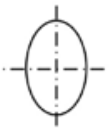

c)

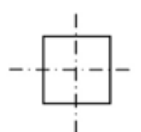

d)

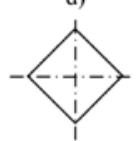

e)

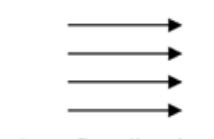

Cross flow direction

Figure 3: Nozzle configurations: a) Circular, b) Elliptic low aspect ratio, c) Elliptic high aspect ratio, d) Square, e) Diamond-shaped

layer are thick. Other parameters of importance for mixing in JICF are the turbulence levels and the confinement of the jet [12] or the nozzle shape $[4,13,14]$.

The extent of a study of turbulent mixing in JICF depends on tools which are capable of probing turbulent scalar fluxes. This study follows a dual approach with both experiments and numerical simulation. The experiments include simultaneous particle image velocimetry (PIV) and laser-induced fluorescence (LIF) which can capture velocity and scalar fields at the same time instants. From such data the turbulent scalar fluxes can be determined without the need of using any model. Numerically, large eddy simulation (LES) provides the same capabilities. A further advantage of LES is that large-scale unsteady coherent structures and their impact on mixing can be tracked accurately in space and time.

This study is focussed on the possibility to influence the highly sensitive flow by the nozzle shape. Such studies have been carried out using PIV, LIF, and Mie scattering [4, 13, 14]. However, this study seeks to further elucidate the JICF exploiting the advantages of LES and simultaneous PIV/LIF described above. Su and Mungal [15] applied simultaneous PIV/LIF to study circular JICF, but did not investigate non-circular nozzle geometries.

It is well-known that mixing in free jets in quiescent atmosphere (no crossflow) can be passively controlled by the aspect ratio (AR), sharp edges, or boundary layer thickness [2]. It is also known that mixing is strongly elevated for JICF as compared to free jets. The near-field entrainment is enhanced for the case with crossflow as there is recirculation 


\begin{tabular}{|l|r|c|c|}
\hline Nozzle Shape & \multicolumn{2}{|c|}{ Dimensions $[\mathrm{mm}]$} & Aspect ratio \\
\hline & Length & Width & \\
\hline Circular & 5 & 5 & 1 \\
\hline Elliptic low AR & 6.25 & 4 & 1.56 \\
\hline Elliptic high AR & 4 & 6.25 & 0.64 \\
\hline Square & 4.43 & 4.43 & 1 \\
\hline Diamond-shaped & 6.26 & 6.26 & 1 \\
\hline
\end{tabular}

Table 1: Nozzle configurations

behind the jet. Secondly, far-field entrainment may be enhanced due to the counterrotating vortices. It is therefore a relevant question if passive control by changing the nozzle geometry is also effective for JICF. The nozzle shapes which are used in the present study are sketched in Figure 3 and their geometric data summarized in Table 1. The nozzles are mounted flush, i.e. the nozzle exit is in the wall plane; their edges are not chamfered. All nozzles have the same cross-sectional area. The following plots are therefore normalized by the diameter of the circular nozzle. Non-circular nozzles always introduce smaller scale vortices into the flow compared to circular nozzles as they have locally higher curvature or even sharp nozzle edges. This enhances turbulent diffusion of momentum and passive scalars. The aspect ratio (AR) has been identified as a second parameter of importance $[4,13,14]$. It is defined as the ratio of spanwise $(W)$ to streamwise $(Q)$ dimensions $(A R=W / Q)$. Elliptic nozzles with high AR have their minor axis aligned with the crossflow direction. The high AR and low AR elliptic nozzles are really the same nozzle, but rotated by $90^{\circ}$. Likewise, the diamond-shaped nozzle is a square nozzle, rotated by $45^{\circ}$. The blunt square and diamond-shaped nozzle have identical $\mathrm{AR}(\mathrm{AR}=1)$ and have the same geometrical shape. The rotation of $45^{\circ}$ therefore only changes the bluntness of the nozzle as seen in crossflow direction.

Liscinsky, True, and Holdeman [14] compare mixing effectiveness of circular, square, elliptic, and rectangular nozzles using Mie-scattering. They find that low AR nozzles form stronger CVPs. This enhances entrainment and gives the passive scalar distribution a kidney shape. However, they argue that the enhanced entrainment is counterbalanced by the smaller jet-crossflow upstream interface, which decreases entrainment. The mixing performance of all nozzles is found to be similar in their study. Haven and Kurosaka [13] measure scalar mixing and visualize vortical structures in a water tunnel by LIF and PIV for elliptic and rectangular nozzles with high and low AR. They show that different vortical structures appear for low and high AR. Moreover, they advocate a lower jet trajectory of their square nozzle compared to their circular nozzle whereas Liscinsky et al.[14] claim the opposite.

The objective of this work is to characterize the mixing performance of various nozzle shapes and orientations and to elucidate the role which coherent structures play with respect to mixing. Section 2 describes the water channel facility and the simultaneous PIV/LIF technique to record the turbulent fluxes. Section 3 describes the governing equations and their boundary conditions and the numerical techniques employed to tackle the problem. The results are presented in Section 4. The main findings are that the global mixing performance is best for the elliptic nozzle with high AR and the blunt square nozzle. The flow fields for these nozzles contain strong modes in transverse direction, enhancing mixing. Additionally, the presence of these modes leads to qualitative differences in the spatial distribution of a passive scalar in a distal (far from the nozzle) cross-sectional plane. The distribution can be single- or double-peaked. Moreover, it is shown that the mean 


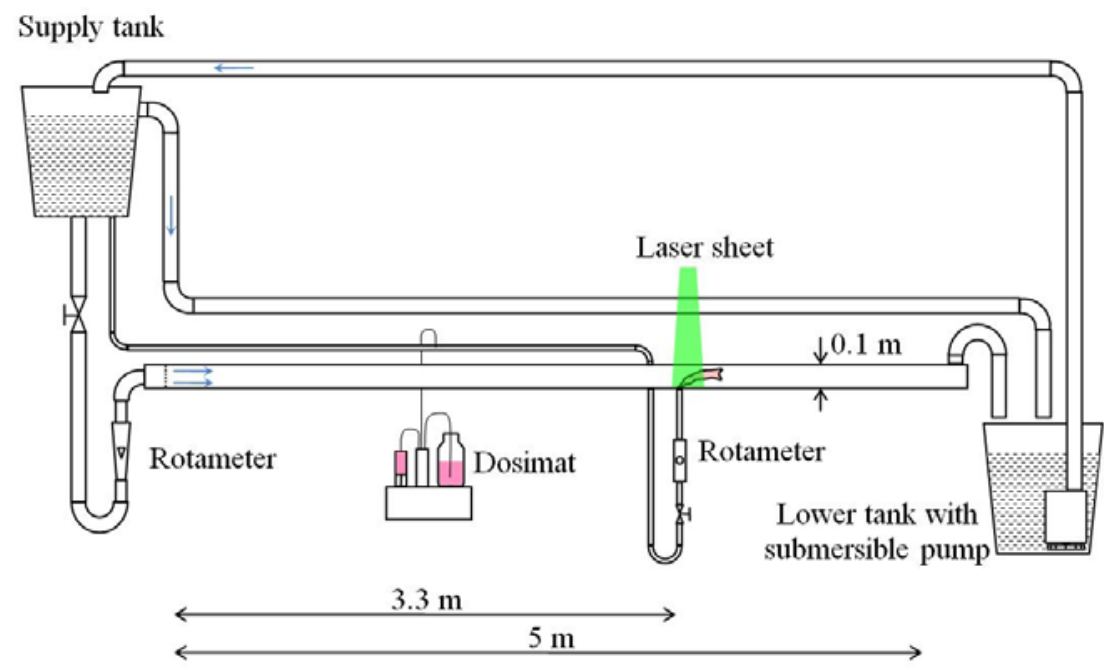

Figure 4: Schematic of the water channel facility

transport of a scalar is against the direction of the mean gradient in important regions of the flow field. In these regions the computed eddy viscosity is negative, whereas turbulence models relying on the gradient diffusion hypothesis typically assume it to be positive.

\section{Experimental Setup and Diagnostic Methods}

\subsection{Water Channel Facility and Experimental Conditions}

The experiments have been carried out in a rectangular $0.1 x 0.1 x 5 \mathrm{~m}$ water channel. A sketch of the entire recirculating water channel facility is presented in Figure 4. The water channel is made of plexiglass to provide optical access over the entire channel length. A transverse jet enters the channel through a tiny nozzle at a normal angle relative to the main flow direction. The diameter of the circular nozzle is $5 \mathrm{~mm}$, resulting in a ratio of channel width to nozzle diameter of $L / D=20$. The jet injection point is 33 channel width $(3.3 \mathrm{~m})$ downstream of the channel inlet and 17 channel width upstream of the channel outlet. A perforated plate to damp secondary motion, a grid to promote fast development of turbulence, and a long section before the jet injection are used so as to promote the development of the turbulent flow in the channel. Nevertheless, the upstream section is still too short to ensure a fully developed channel flow. This fact can be observed in the velocity profiles and the spectral content of the turbulence fluctuations. These secondary flow effects are, however, small as compared to the structures due to the transverse jet in cross-sectional planes in the wake of the jet. The main channel flow and the jet flow are gravity driven from an upper tank with overflow system. The volumetric flow rates for the crossflow and the jet are regulated independently by valves and measured using rotameters. The inaccuracy of the volume flow measurements is about $3 \%$ of the full scale. The Reynolds number of the crossflow based on the channel width is $R e=10000$ which corresponds to a bulk velocity of $0.1 \mathrm{~m} / \mathrm{s}$. The recirculating water is at room temperature $\left(20^{\circ} \mathrm{C}\right)$. The velocity ratio based on bulk velocities is $R=\frac{V_{\text {jet }}}{V_{\text {crossflow }}}=4$. 
High pass filter

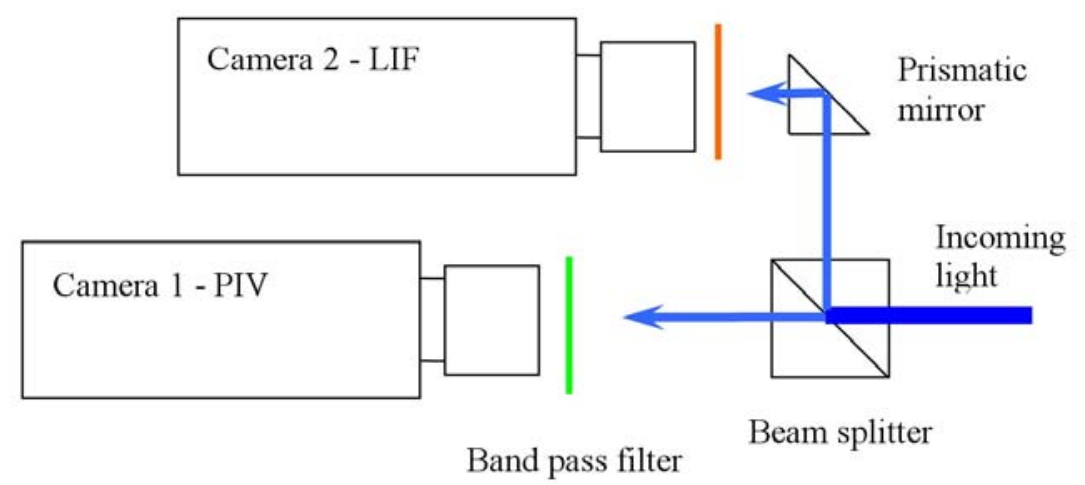

Figure 5: Layout of the camera arrangement for simultaneous PIV/LIF measurements

\subsection{Simultaneous PIV and LIF}

PIV is based on determining the displacement of tracer particles after a short time interval. Two short pulses of a thin laser sheet generated by a double cavity Nd-YAG laser illuminate the particles; these in turn scatter light which is collected by a CCD (charge-coupled device) camera. The present laser system produces two pulses with a duration of $7 n s$ each and an energy of $25 \mathrm{~mJ}$ per pulse. The interval between the two pulses of a double pulse in this study is set to $500 \mu \mathrm{s}$. By passing the laser beam through a set of cylindrical and collimating lenses, a laser sheet is created with a thickness of $1.5 \mathrm{~mm}$. The seeding for the PIV measurements consists of hollow glass spheres with a nominal diameter of $10 \mu \mathrm{m}$ and a density of $1000 \mathrm{~kg} / \mathrm{m}^{3}$. These have sufficient size to scatter enough light but are also small enough to follow the flow accurately. The Stokes number, St, of the glass spheres is on the order of $S t \sim 10^{-4}$. Seeding for the crossflow is injected into the tank; the seeding for the jet is injected (together with the fluorescent dye for LIF) 250 jet nozzle diameters upstream of the jet injection point. The long channel (33 channel widths) with flow straighteners before the test section ensure even distribution of the tracer particles (and the dye for LIF). The concentration of tracers in the jet is kept constant by maintaining a steady injection rate with the use of an electrical syringe.

The same laser sheet excites Rhodamine B which is the tracer species for LIF. When the dye de-excites, it emits light at a longer wavelength. The signal intensity is proportional to the local tracer concentration if signal quenching has a small effect. The linear range of LIF is determined by a calibration curve. The fluorescent light is captured by a second CCD camera. Rhodamine B is an efficient tracer because it has peak absorption at a wavelength of $540 \mathrm{~nm}$, which is close to the laser wavelength $(532 \mathrm{~nm})$, and peak emission at $625 \mathrm{~nm}$.

One can use a single laser sheet for PIV and LIF measurements since the PIV and the LIF signals can be separated by their wavelengths. The light coming from the test section, containing both the PIV and the LIF signal, is divided into two signals by a beam splitter, as sketched in Figure 5. The beam splitter is a glass cube made of two triangular prisms with a thin silver coating on the joint surface. When light hits this surface, one half is reflected and the other half is transmitted. The two signals are then caught by two identical CCD cameras. In order to separate the PIV signal from the LIF signal, each camera is equipped with a filter for different wavelength. The PIV camera has a band pass optical filter $(532 \pm 3 \mathrm{~nm})$ whereas the LIF camera has high band pass filter made of OG 
550 material with cut-off wavelength of $550 \mathrm{~nm}$ and maximum transmission at $625 \mathrm{~nm}$.

The cameras are equipped with $60 \mathrm{~mm}$ imaging lenses with an aperture of $f=2.8$. The pixel resolution is $1280 x 1024$ and the dynamic range is 12 bit. Using a calibration plate the cameras are adjusted so that the dislocation between images is at most 2 pixels. The camera field of view is about $63 x 50 \mathrm{~mm}$ which corresponds to a spatial resolution of $50 \mu \mathrm{m}$ per pixel in both vertical and horizontal directions. The PIV camera takes images in double frame/double exposure mode which enables cross-correlation. The final interrogation window is set to 16 pixels. Since the concentration of seeding is high, individual particles cannot be tracked. The image is divided into interrogation windows and average particle displacements are obtained by cross-correlating between interrogation windows of image pairs. To achieve higher accuracy, the sub-pixel displacement is fitted with a threepoint Gaussian peak. The correlation peak results in an estimated displacement down to 0.1 pixels. The LIF camera is set to single frame/single exposure mode with a CCD chip exposure time of $800 \mu s$.

\subsection{Error Estimates of the Measurements}

The mass flow rate of the jet has an uncertainty of $3 \%$, as could be estimated from the time to fill a bucket with known volume. The same uncertainty is estimated for the crossflow (main channel) flow rate. The PIV has an estimated error of $2 \%$ based on minimal detectable displacement of 0.1 pixels. The number of recorded PIV images is 400. The statistical uncertainty for the mean is up to $3 \%$ in the shear layer and up to $1 \%$ elsewhere, and for the rms below $8 \%$ in the shear layer and $3 \%$ elsewhere.

Since the laser sheet illumination is not even, images with uniform concentration are taken and used for calibration. The final concentration distribution is calculated by subtracting the background image from the instantaneous image and normalizing it with the calibration image. The intrinsic uncertainties of the LIF method stem from shot-to-shot variations and non-uniformities in the laser sheet illumination, background noise, and uncertainty in the preparation of the fluorescent dye solution. The LIF signal intensity is proportional to the LIF tracer concentration within 3\%. Background noise is $4 \%$ of the maximum signal intensity. The estimated error for the non-uniformities in the laser sheet is about $6 \%$. The LIF estimate is based on the distribution of scalar in the potential core, which we expect to be uniform. The estimated error of the LIF technique is about $13 \%$.

\section{Large Eddy Simulations}

\subsection{Governing Equations}

The governing equations are the Navier-Stokes equations for incompressible flow. Additionally, mixing is studied by integrating a transport equation for a passive scalar. In LES, the equations are filtered to eliminate the small scales from the flow field, but thereby maintain the large scales. This is done with a filter function $F$ according to Eq. 3. The filter function $F$ has a characteristic filter scale, $\Delta$. The filtering implies that the filtered variable $\widetilde{\phi}$ does not contain Fourier components that are shorter than $\Delta$.

$$
\widetilde{\phi}\left(x_{0}, t\right)=\int_{-\infty}^{\infty} F\left(x-x_{0}\right) \phi(x, t) d x
$$

The filtered Navier-Stokes equations are Eq. 4 and Eq. 5. Similarly, the filtered transport equation for a passive scalar is Eq. 6. 


$$
\begin{gathered}
\frac{\partial \tilde{u}_{i}}{\partial x_{i}}=0 \\
\frac{\partial \tilde{u}_{i}}{\partial t}+\tilde{u}_{j} \frac{\partial \tilde{u}_{i}}{\partial x_{j}}=-\frac{\partial \tilde{p}}{\partial x_{i}}+\frac{1}{R e} \frac{\partial^{2} \tilde{u}_{i}}{\partial x_{j}^{2}}-\frac{\partial}{\partial x_{j}}\left(\widetilde{u_{i} u_{j}}-\tilde{u}_{i} \tilde{u}_{j}\right) \\
\frac{\partial \tilde{c}}{\partial t}+\tilde{u}_{j} \frac{\partial \tilde{c}}{\partial x_{j}}=\frac{1}{R e S c} \frac{\partial^{2} \tilde{c}}{\partial x_{j}^{2}}-\frac{\partial}{\partial x_{j}}\left(\widetilde{c u_{j}}-\tilde{c}_{j}\right)
\end{gathered}
$$

$\frac{\partial}{\partial x_{j}}\left(\widetilde{u_{i} u_{j}}-\tilde{u}_{i} \tilde{u}_{j}\right)$ and $\frac{\partial}{\partial x_{j}}\left(\widetilde{c u_{j}}-\tilde{c} \tilde{u}_{j}\right)$ are the subgrid-scale (SGS) turbulent stresses and SGS turbulent fluxes, respectively. Re is the Reynolds number of the flow and $S c$ is the Schmidt number of the dye.

\subsection{Subgrid-Scale Modeling}

In LES, the large-scale eddies are resolved while the universal small-scale eddies are described by SGS models for turbulent stresses and turbulent scalar fluxes. There is several modeling approaches for the small-scale eddies available, for example the Smagorinsky model [16], Scale Similarity Model [17], the Dynamic Model [18], or the so-called implicit SGS model, i.e. no explicit SGS model, e.g. [19]. The latter is chosen in the present work for the following reasons. The modeling assumption in the implicit model is that the unresolved scales contain little energy which can therefore be neglected. SGS models describe the physical processes on the border between resolved and unresolved scales. The most important is to drain kinetic energy from the large scales which is dissipated at the smallest scales of turbulence (in the real, physical world). Any stable numerical scheme can be used for this draining of the kinetic energy from the large scales, and this leads to the idea to use no explicit turbulence model. Secondly, the effect of any SGS model must always decline with increasing resolution, since the filtered Navier-Stokes equations will return the unfiltered Navier-Stokes equations in the limit of zero filter size. It follows immediately that increasing the resolution in LES will give resolution of eddies of decreasing size, until the dissipative eddies are resolved for sufficiently fine grids which are frequently used as implicit filter. Under such conditions the computations become so-called direct numerical simulation (DNS): In this respect LES is an approximation to DNS, as opposed to situations in which the model term does not vanish with complete resolution. Conceptually, this has the implication that there is no need for any turbulence model if just the grid is fine enough. Increasing the spatial resolution leads to increasing the separation of scales between the largest eddies and the unresolved eddies. Increasing the resolution leads also to better resolution of the so-called backscatter, the intermittent transfer of energy from the small scales to the large scales. Physical backscatter refers to the fact that intermittently vortices can increases in scale, e.g. by merging with another vortex or by an adverse pressure gradient in the axis parallel direction. These phenomena are obviously resolved better on finer grids (as opposed to the numerical backscatter of discretization schemes). The ever increasing computer power therefore increases the level of approximation of LES, but especially of the implicit turbulence model. Additionally, it has been demonstrated that the discretization error of numerical schemes is on the same order as the computed SGS flux in a wide range of resolvable waves [20,21]. This means that numerical schemes intrinsically interact with any turbulence model unless there is separation of scales between the grid size and the filter size. It can therefore be suggested that it may not be beneficial to use an explicit SGS model [22, 23]. 


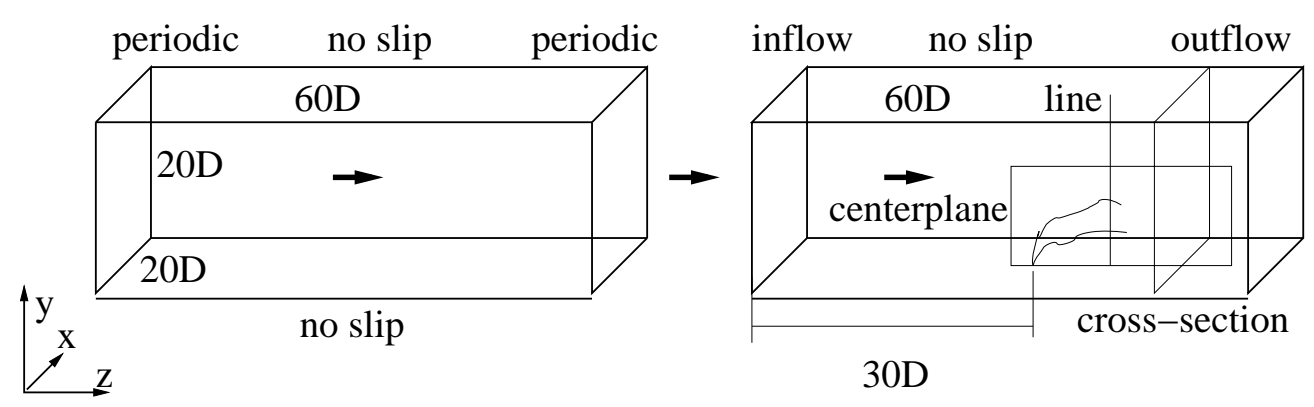

Figure 6: Geometry for computational problem

\subsection{Numerical Methods}

The spatial discretization of the convective terms is done with a fifth-order weighted essentially non-oscillatory (WENO) scheme [24] to handle the strong streamline curvature near the jet injection. The diffusive terms are discretized with fourth-order central differences. The Navier-Stokes equations are integrated in time explicitly with a third-order Runge-Kutta scheme. Pressure corrections are obtained from a Poisson equation which is relaxed with a pointwise Gauss-Seidel iteration scheme. The convergence is accelerated by the multigrid method [22]. The transport equation for the passive scalar is also integrated with these high-order methods. The staggered grid is stretched to improve the near-wall resolution. On the finest grid, $y^{+} \sim 3$, which requires about 3.8 million cells.

\subsection{Boundary Conditions}

The geometry of the computed problem is presented in Figure 6. The upper and lower walls have no-slip boundary conditions for the velocities. Periodic conditions are applied in the lateral direction. The channel is divided into two parts: The first half simulates an infinitely long channel by using periodic boundary conditions in streamwise direction. This channel provides the inflow boundary condition for the second part of the channel. The velocity outflow boundary condition for the second half of the channel is a fluxconservative zero-gradient condition. The inflow and outflow boundaries are far from the region of interest, and thereby their impact on the result is decreased. The scalar is set to zero at the walls in the base case, except in the nozzle. The lateral direction is assumed to be periodic, the inflow condition is zero for the scalar, the outflow a zero-gradient.

\subsection{Resolution Issues of the Nozzle Geometry}

The geometrical and flow details that are smaller than the filter size are unresolved. Thus, they are handled consistently by applying a filter (Eq. 3) to it. This aspect is important in this particular case, since the geometrical shape of the jet nozzle may include scales that are smaller than those supported by the current LES. This approach differs from the LES computations of Yuan, Street, and Ferziger [1], in which the nozzle is not only well resolved, but also the pipe from which the jets exits is meshed upstream. For the channel flow velocity profile, on the other hand, they prescribe a parabolic velocity profile. In the present study, the channel flow is fully developed turbulent due to the periodic part of the channel as described above. The nozzle geometry itself is described in a local two-dimensional grid with a cell size 25 times smaller than the LES grid cell size $\left(h_{\text {geometry }} / h_{L E S}=1 / 25\right)$. One the LES grid, the nozzle diameter is then resolved by $3-4$ 


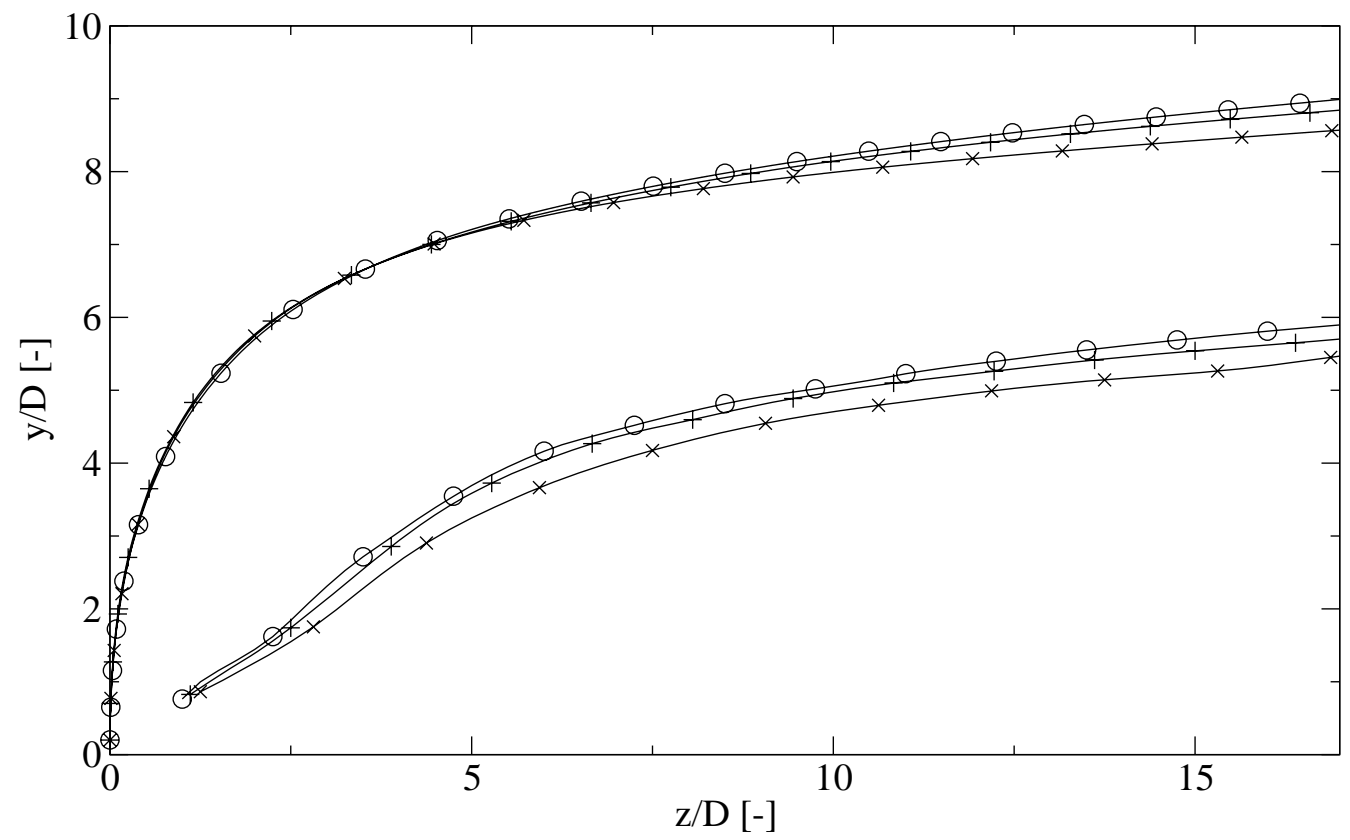

Figure 7: Numerical accuracy of the trajectory based on the mean streamline and the vortex curve, million cells: $\bigcirc-3.8,+-3.2, \mathrm{x}-2.4$

cells. In this study, the velocity profiles are set to be top-hat profiles at the geometrical nozzle location with a non-dimensional velocity of four, so as to simulate a JICF with a velocity ratio of four. This particular velocity ratio was chosen since the wake vortices (see Figure 1) are strongest in this velocity ratio range [7]. The scalar is set to unity at the same place. Consistent with the LES methodology, one can compute filtered nozzle boundary conditions simply by applying Eq. 3 at the boundary. In this case a Gaussian filter kernel with a filter scale equal to a single cell on the LES grid is applied.

\section{Results}

\subsection{Flow Field of a JICF}

In numerical studies one must judge the numerical accuracy of the computed results by computing a solution on several grids. As discussed in Section 1, the trajectory of the deflected jet is a parameter of paramount importance with respect to mixing. One can define several characteristic curves for a JICF which have been called trajectory $[15,25]$. Two such curves are the mean streamline from the center of the nozzle and the so-called "vortex line", the locus of points with maximum upwash between the two vortices of the CVP, following Fearn and Weston [26]. Figure 7 characterizes the JICF in terms of these characteristic lines. One notes that the CVP is initiated in the wake of the deflected jet and lies significantly lower than mean streamline. The solutions for the mean streamline and the CVP are computed on three grids with 2.4, 3.2, and 3.8 million cells. The finer the grid, the higher the curves become. The three solutions for the lift-off height lie within $5 \%$. Note that the filter width is proportional to the cell size and therefore also smaller eddies are resolved on finer grids. Figure 7 shows therefore the sensitivity to the cell size under these conditions. For a study of purely numerical accuracy in the framework of LES, one needs a scale separation (order of magnitude) between the filter size and the cell size. This is of course not feasible at present as the computer power is excessive [27]. 


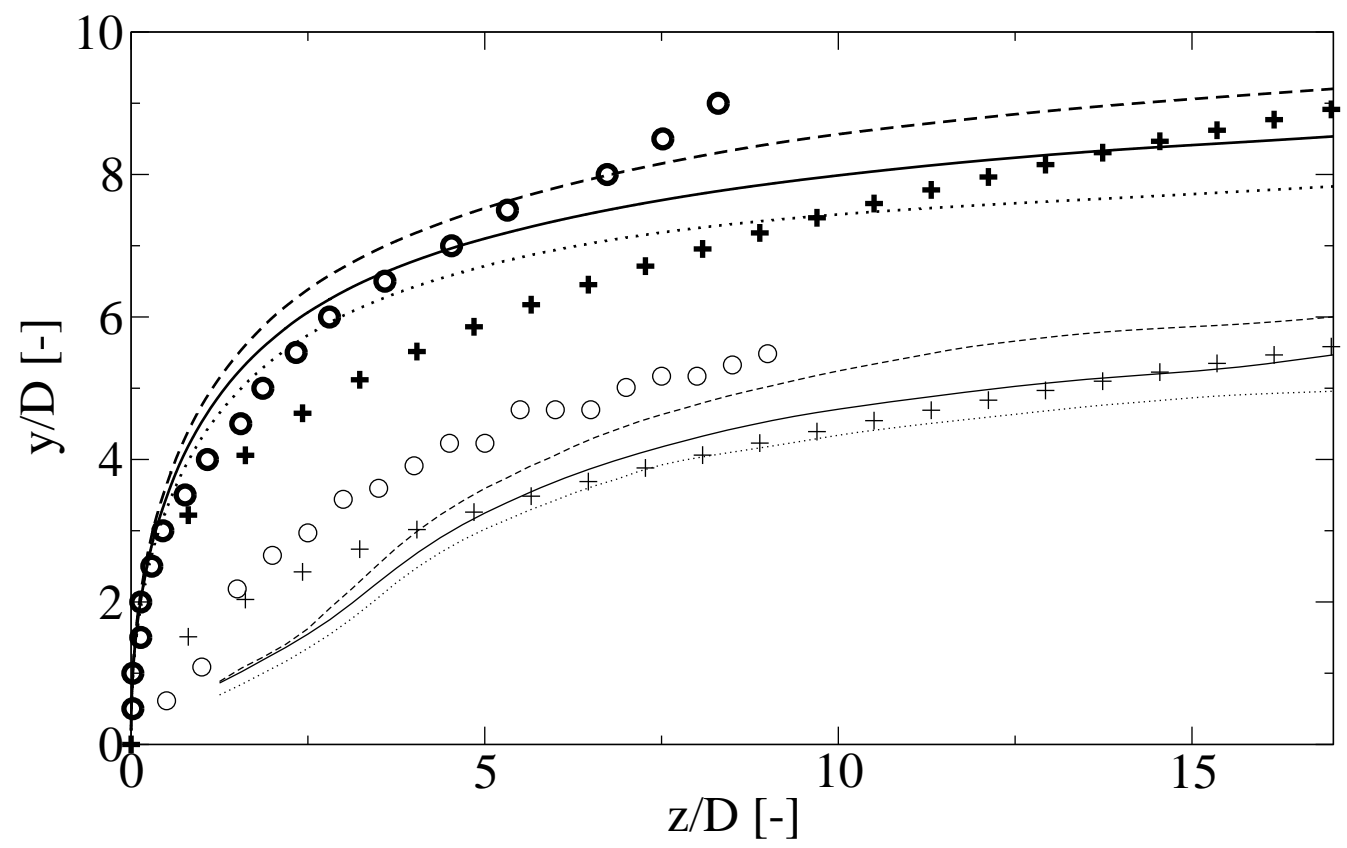

Figure 8: Comparison of the computed $(\cdots-\mathrm{R}=3.75$, $--\mathrm{R}=4,---\mathrm{R}=4.25)$ and measured (O) trajectories with measurements by Fearn and Weston [26] (+), upper curves for each symbol (thick): Mean streamline, lower curves (thin): Vortex curve

The trajectory data in the literature shows significant spread, which only partly can be attributed to varying definitions [25]. Other important factors are boundary layer thickness, jet velocity profile [11], geometric differences [12] and turbulence levels. In Figure 8 the measured and computed data of the present study is compared to the data of Fearn and Weston [26]. The upper curves (fat print) of each symbol are the mean streamlines. The lower (thin print) curves are the vortex curves. An issue which has not received much attention is the accuracy with which the nominal conditions can be realized. Figure 8 compares the computed solution for small changes in mass flows. If the mass flows of the jet and the crossflow can be ensured with $3 \%$ accuracy each (estimated by a bucket test), then the actual velocity ratio lies between $R=3.75$ and $R=4.25$ for a nominal velocity ratio of $R=4$. The trajectory height then varies by about $15 \%$ in the far-field. This variation is also compared to the spread in measured data in Figure 8, which includes the present PIV measurement and the measurement of Fearn and Weston as examples [26], which also has a velocity ratio of $R=4$. The difference between the present measurements and the measurements by Fearn and Weston [26] is typical for the scatter in the trajectory data for different setups. This scatter in the experimental data makes sensitivity analysis of computed results more important than the exact values of the computed results themselves. The differences in the computed curves and the two independent measurements for nominally identical conditions show the strong sensitivity of the flow field to possible sources of discrepancies, e.g. secondary flow features or the above mentioned factors which are known to have strong impact on the trajectory heights.

Figure 9 displays profiles of $v$ along the cross-stream line (see Figure 6 ) at $z / D=10$ as computed on the three grids. The line lies in the centerplane between the two vortices of the CVP. The velocity maximum at $y / D \approx 5$ is not attributed to the jet but marks the upwash between the two vortices of the CVP. The maxima in Figure 9 lie on the vortex curves in Figure 7. Their magnitude hence characterizes the strength of the average CVP. The upwash varies by about $15 \%$ for the three grids, but only by $5 \%$ for the two finest grids. A corresponding observation can be made in Figure 10 from which the numerical 


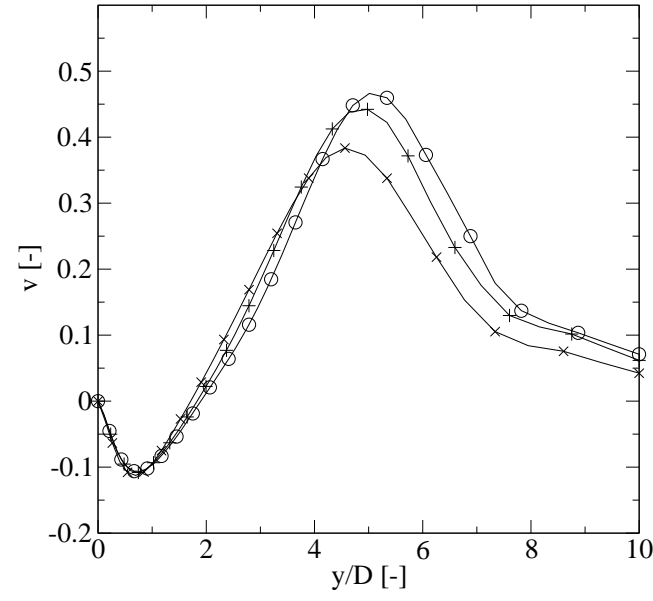

Figure 9: Numerical accuracy mean $\mathrm{v}$, million cells: $\bigcirc-3.8,+-3.2, \mathrm{x}-2.4$

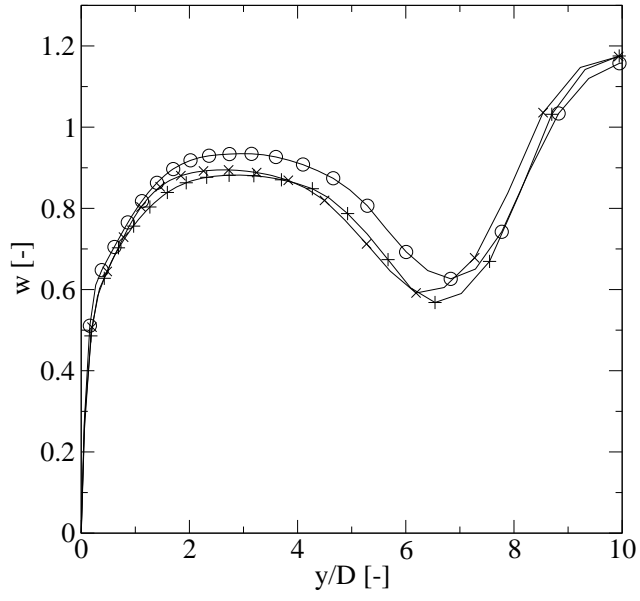

Figure 10: Numerical accuracy mean w, million cells: $\bigcirc-3.8,+-3.2, \mathrm{x}-2.4$

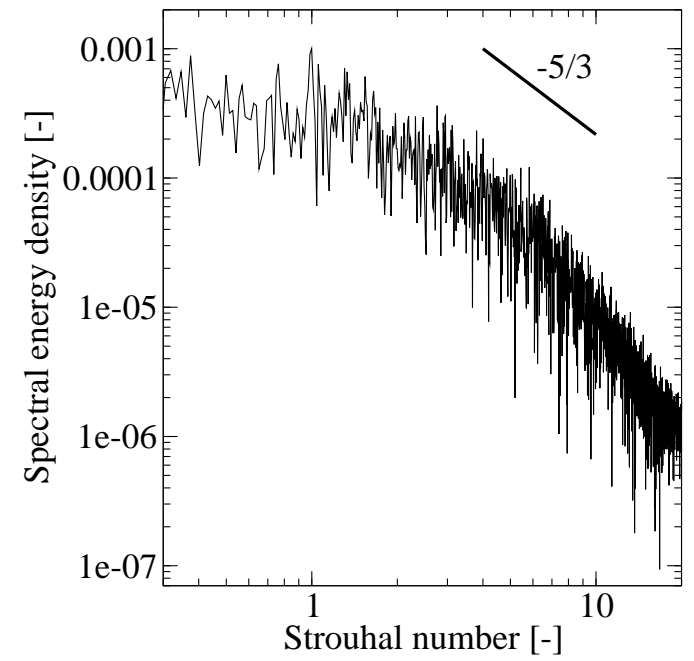

Figure 11: Turbulent kinetic energy spectrum in the centerplane at $y / D=5$ and $z / D=10$ 


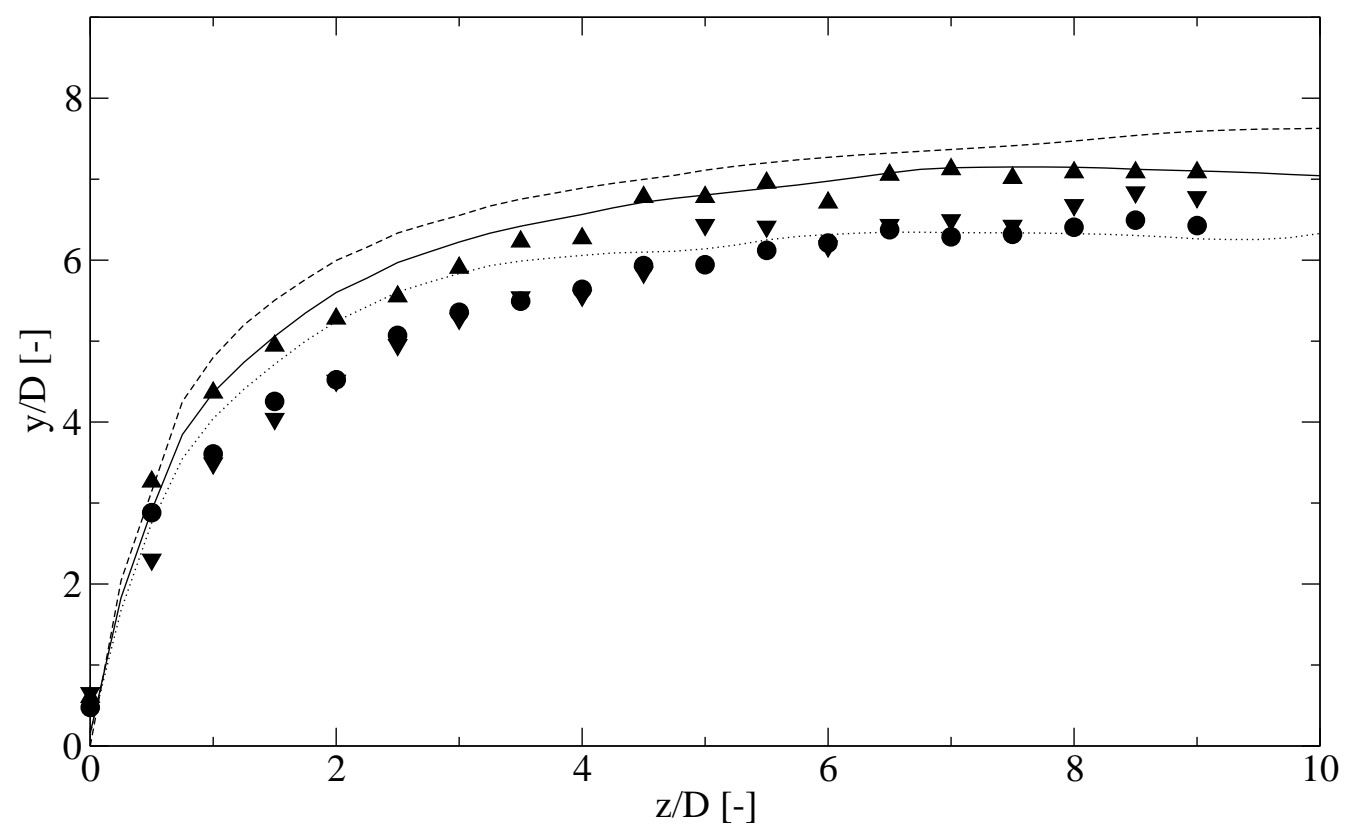

Figure 12: Computed (c) and measured (m) mean c trajectories for various nozzle shapes: Round $(\mathrm{m}) \bigcirc$, round $(\mathrm{c}) —$, elliptic low $\mathrm{AR}(\mathrm{m}) \triangle$, elliptic low AR (c) - - - , elliptic high AR $(\mathrm{m}) \nabla$, elliptic high AR (c) ...

accuracy for the streamwise velocity component at $z / D=10$ can be estimated. The jet upstream manifests in the momentum deficit compared to a full turbulent channel flow velocity profile. The velocity profile has a minimum at $y / D \approx 6-7$. The minimum stems from the region of highest momentum exchange between the jet and the crossflow (the region of highest jet trajectory curvature). The solution varies by about $10 \%$ for the streamwise velocities at this location for the three grids, and by about $3 \%$ for the two finest grids.

Figure 11 shows the spectral content of the flow in the wake in the centerplane at $y / D=5$ and $z / D=10$. This is approximately on the vortex line (see Figure 7 ). Sufficient parts of the inertial subrange are resolved as can be seen by comparison with the theoretical slope of $-5 / 3$ for fully developed turbulence. The two largest peaks have Strouhal numbers of $S t=1$ and $S t=0.4$, respectively. This is different from the Strouhal number behind a solid cylinder $(S t=0.2)$. This also shows that wake vortices are fundamentally different from the von Karman vortex street [7].

\subsection{Penetration of a Scalar into the Crossflow}

A third characteristic curve, that has frequently been used to characterize the penetration of the jet into the crossflow, is the locus of points with maximum mean scalar concentration in the symmetry plane. This curve can be computed from LES data and from the LIF measurements. The global maxima of the scalar may lie, however, off the centerplane [28]. They may be as much as one nozzle diameter below the penetration implied by the maxima in the symmetry plane [25]. This is due to the large-scale mixing effect of the CVP. If one compares the relative positions of the CVP (Figure 8), here defined as the locus of points with maximum upwash between the two vortices, and the scalar peaks (Figure 12), one notes that the scalar peaks are clearly above the CVP. The CVP counter-rotates in a sense that fluid above the CVP is drawn laterally away from the symmetry plane. Morevoer, the lateral transport of the scalar is dependent on the nozzle geometry as is discussed in Section 4.3. 


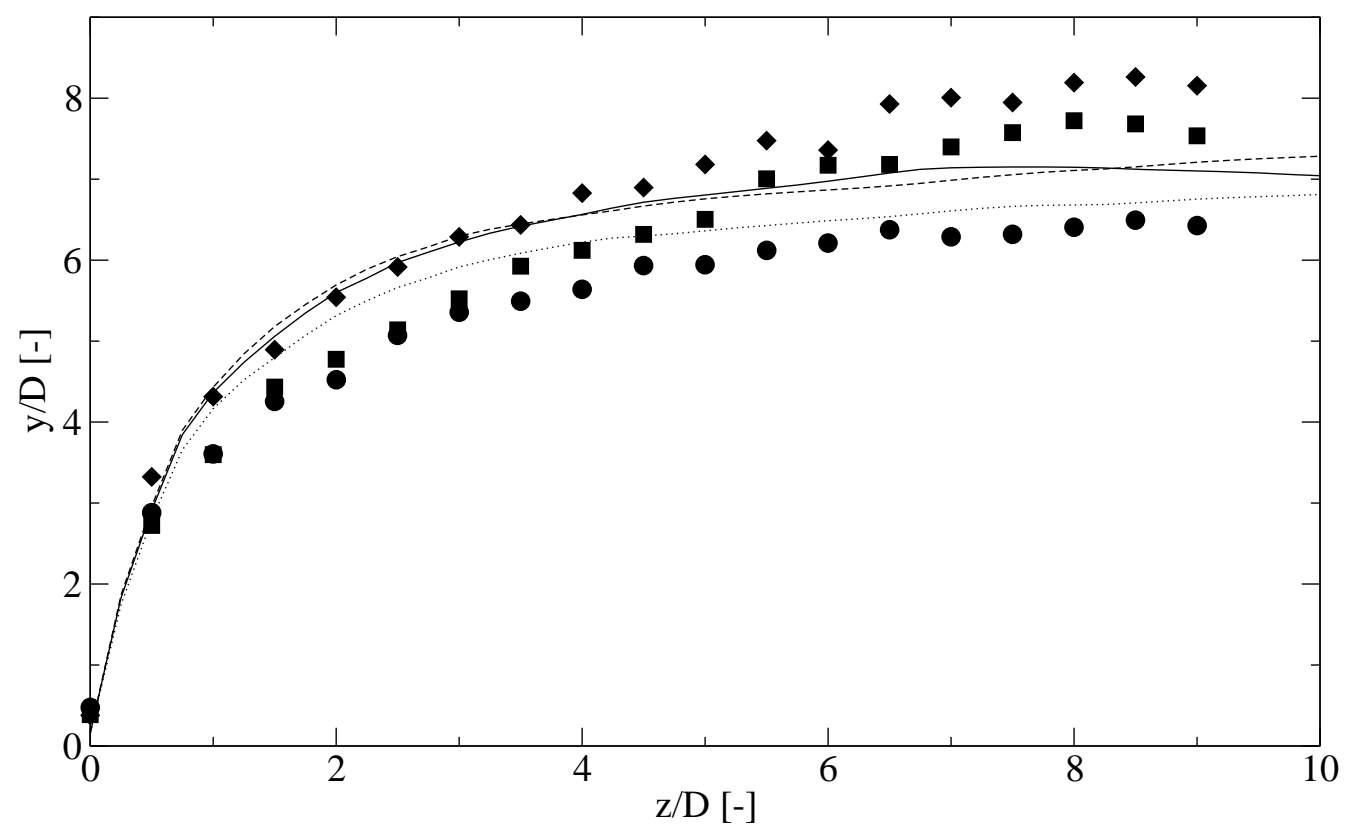

Figure 13: Computed (c) and measured (m) mean c for various nozzle shapes: Round $(\mathrm{m}) \bigcirc$, round $(\mathrm{c})-$, square $(\mathrm{m}) \square$, square $(\mathrm{c}) \cdots$, diamond $(\mathrm{m}) \diamond$, diamond $(\mathrm{c})--$

The effect of the AR on the jet penetration is shown in the computed results in Figure 12: The higher the AR, the lower the jet penetration. This result agrees with the results of Haven and Kurosaka [13] and Liscinsky et al.[14]. In the LIF measurement the trajectory obtained for the circular nozzle does not satisfy this trend. Measuring such differences can be difficult due to the intrinsic uncertainty of LIF and since the flow field is highly sensitive to other small changes in boundary conditions. The agreement between measured and computed results for scalar trajectories is therefore within expected error margins.

Figure 13 addresses the effect of the square nozzles and their orientation. In computations the trajectory for the circular nozzle is above the trajectory for the blunt square nozzle, but the experimental data shows the opposite trend. There is contradictory data in previous published work concerning this comparison: Haven and Kurosaka [13] suggest a larger jet penetration for round nozzle whereas Liscinsky, True, and Holdeman [14] demonstrate a larger penetration for the square nozzle. This is another example of the large spread in the data in the literature due to sensitivity to small changes in boundary conditions, e.g. secondary flow, turbulence levels, boundary layer thickness. In the current experiment and computation, the jet from the blunt square nozzle penetrates less deeply compared to the diamond-shaped nozzle case. As the AR and nozzle shape are identical for both nozzles, the effect of bluntness is clearly revealed: Blunt nozzles have a stronger recirculation zone and produce more turbulence [25]. This decreases the jet penetration.

\subsection{Scalar Spatial Distribution}

Figure 14 shows the spatial distribution of passive scalar in a proximal (near the nozzle) cross-sectional plane at $z / D=4$. The peak is within the isoline $c>0.2$. In this proximal plane there is only one peak which lies in the symmetry plane. The horseshoe shape of the isoline $c=0.1$ is explained by the recirculation zone behind the jet: There is no convective transport from the jet into the direct lee of the jet. Convection transport laterally around the recirculation zone forms the two lobes of the horseshoe. In a distal cross-section at $z / D=12$ (Figure 15) the scalar distribution is double-peaked due to large-scale transport of the CVP. This fact has relevance for interpretation of data obtained in the symmetry 


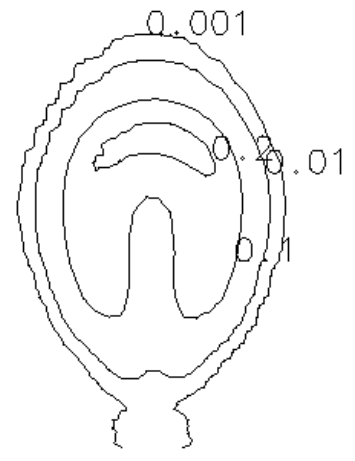

Figure 14: Circular nozzle, proximal crosssectional (xy) plane at $\mathrm{z} / \mathrm{D}=4$

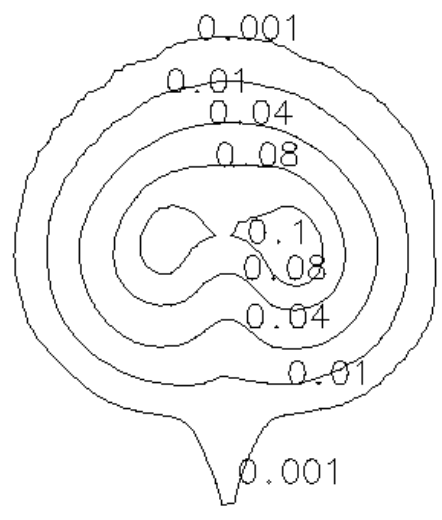

Figure 15: Circular nozzle, distal crosssectional (xy) plane at $\mathrm{z} / \mathrm{D}=12$

plane: It is impossible to decide from views on the symmetry plane alone if the scalar distribution shows a global peak or a saddle point between two global peaks. Threedimensional data is therefore necessary to aid the interpretation of the data. Even more so, since the scalar distribution only becomes double-peaked for larger velocity ratios. At $R_{e}=7.7$, Kamotani and Greber [28] obtain a single-peaked distribution in a proximal plane and double-peaked distribution in a distal plane, whereas at $R_{e}=3.9$ they find single-peaked distribution even in the far-field. The present simulation has a velocity ratio of $R=4$ and a double-peaked distribution. Kamatoni and Greber investigate a non-isothermal jet, and hence the effective velocity ratio $R_{e}$ (Eq.2) characterizes the flow, whereas in this study and in Liscinsky, True, and Holdeman [14] isothermal flows are considered, for which the densities are equal, and $R_{e}$ can be replaced by the real velocity ratio $R$ (Eq.1).

Figure 16 to Figure 19 demonstrate that the nozzle shape has profound effects on the spatial distribution of the passive scalar. The elliptic nozzle with high AR and the blunt square nozzle have only one peak even at the distal location at $z / D=12$. Low AR elliptic nozzles and diamond-shaped nozzles, on the other hand, have two peaks as the circular nozzle. In the Mie-scattering study by Liscinsky, True, and Holdeman [14] at $R=2.9$, the scalar distributions for circular, square, and two elliptic nozzles with $A R=2$ and $A R=0.5$ are single-peaked. This qualitative difference is the result of the competition between small-scale turbulent mixing and large-scale mixing due to the CVP as is suggested below using proper orthogonal decomposition (POD). The nozzles producing one peak have elevated turbulence levels [25]. The CVP and the jet (the mean scalar region) meander with larger velocities in the turbulent flow field. One the other hand, nozzles with lower turbulence levels have dominant large-scale transport due to the CVP, resulting in two peaks. Another distinction lies in shape of isolines. The isolines for the high AR elliptic nozzle have only small portions with concave curvature: They are egg-shaped rather than kidney shaped.

\subsection{Global Mixing Performance}

As it is difficult to address mixing performance from such qualitatively different distributions, reduction of the data to global parameters seems necessary. The mixedness of the flow can be computed for each cross-sectional plane $[14,29]$. The average passive scalar $\widehat{c}$ 


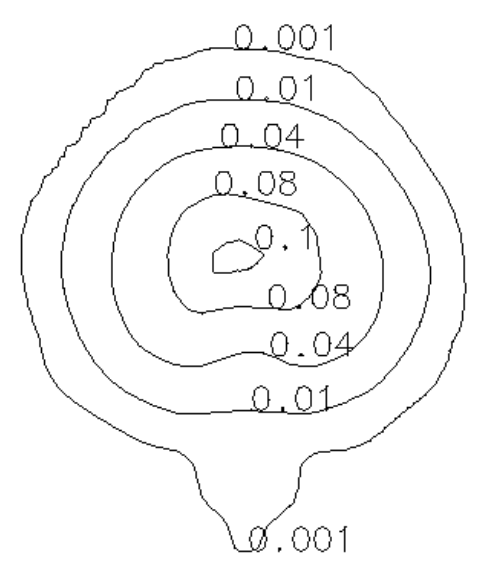

Figure 16: High aspect ratio elliptic, (xy) plane at $\mathrm{z} / \mathrm{D}=12$

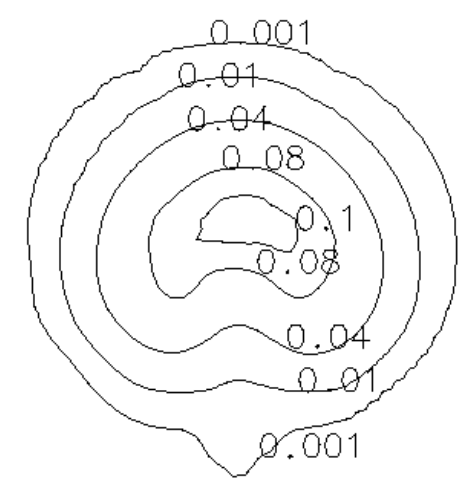

Figure 18: Blunt square, (xy) plane at $\mathrm{z} / \mathrm{D}=12$

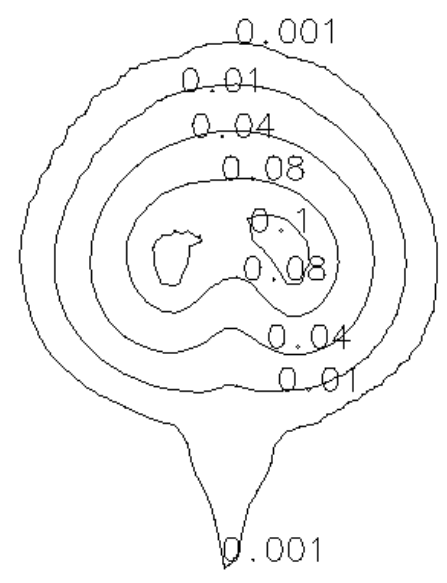

Figure 17: Low aspect ratio elliptic, (xy) plane at $\mathrm{z} / \mathrm{D}=12$

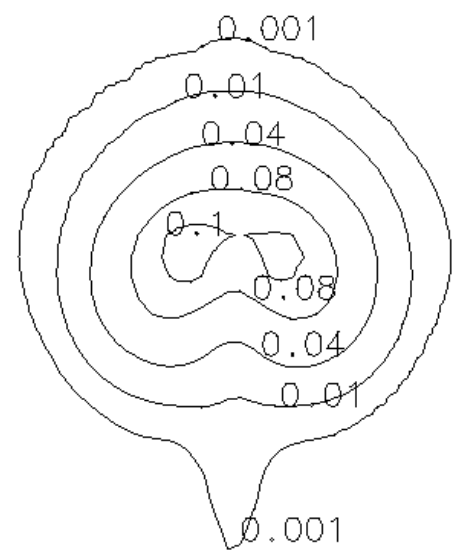

Figure 19: Diamond-shaped, (xy) plane at $\mathrm{z} / \mathrm{D}=12$ 


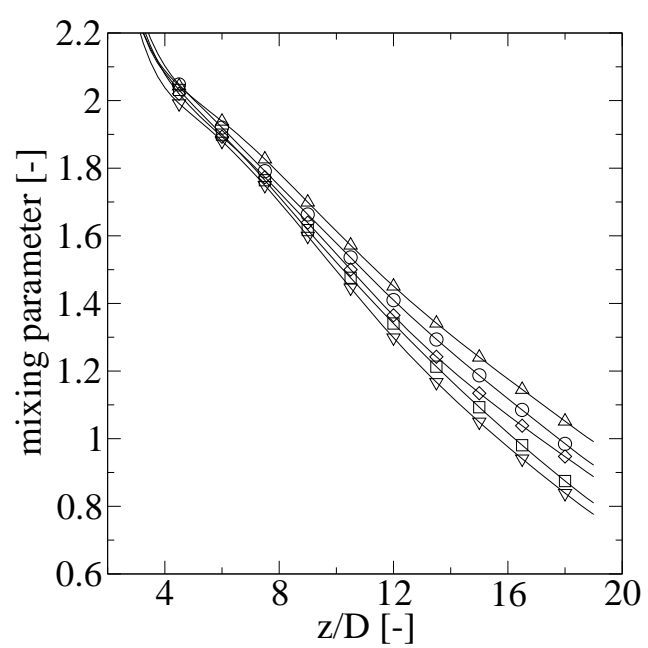

Figure 20: Mixing parameter for various nozzle shapes: $\bigcirc$ - round, $\triangle$ - elliptic low AR, $\nabla$ - elliptic high AR, $\square$ - square, $\diamond$ diamond

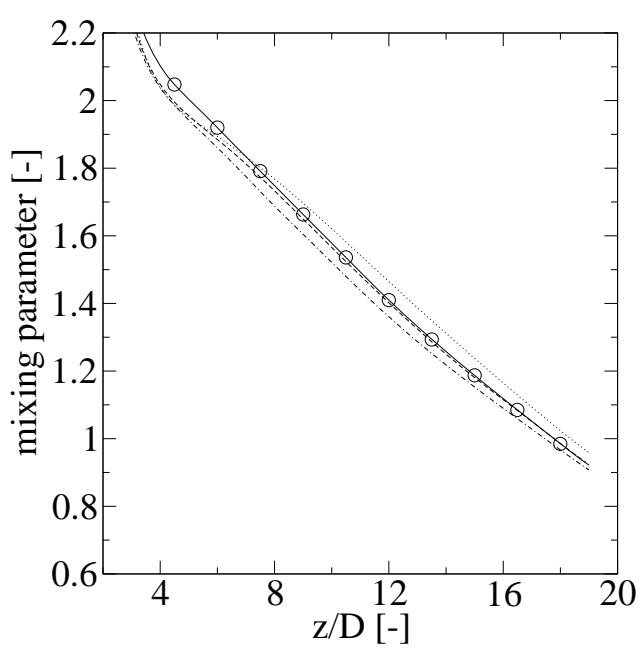

Figure 21: Numerical accuracy for mixing parameter: $\bigcirc-3.8$ million cells, ----3.2 million cells, - - 2.4 millions cells, -·- - zero gradient boundary condition

in a cross-sectional plane is defined by Eq. 7, its spatial variance $c^{\prime \prime}$ by Eq. 8 . The mixing parameter is $M$ defined as the normalized square root of the spatial variance, as given by Eq. 9.

$$
\begin{gathered}
\widetilde{c}=\frac{1}{A} \int c d A \\
c^{\prime \prime}=\frac{1}{A} \int(c-\widehat{c})^{2} d A \\
M=\frac{\sqrt{c^{\prime \prime}}}{\widehat{c}}
\end{gathered}
$$

The smaller the mixing parameter, the more homogeneous the mixture. If the spatial variance and hence the mixing parameter are zero, the mixture is perfectly homogeneous. The mixing parameter is plotted as function of distance from the nozzle in Figure 20. The ordinate of the curve is a measure of the heterogeneity whereas the slope measures the mixing efficiency. The magnitudes are obtained for an integration area of $8 D x 7 D$, which includes all regions with $c>0.01$ for all nozzles. The difference in mixing parameter is up to $23 \%$ for the given integration area. Since the mixing parameter depends on the integration area, not only the absolute value but also the magnitude of the relative change are immaterial. The order of the mixing performance of the nozzles is of course independent of the integration area.

Nozzles which have single-peak distribution of the scalar are clearly better mixers than nozzles which produce a double-peak. Liscinsky et al. [14] find smaller differences of the mixing performance at $R_{e}=2.9$. However, at this effective velocity ratio all distributions are single-peaked as their scalar distributions show. Comparing the elliptic nozzles to the circular nozzle, one notes two effects: First, the higher the AR, the better the mixing. Second, both elliptic nozzles are identical in shape and both introduce smaller scale vortices into the flow, improving small scale mixing. For high AR the improved mixing due to shape adds to the improved mixing due to AR, resulting in large differences in mixing parameters between the high AR elliptic and the circular nozzles. For low AR, on the other hand, improved mixing due to smaller scales competes with the deteriorated 
mixing due to AR, and the difference between curves for the low AR and circular nozzles is smaller as compared to the difference between the curves for the high AR elliptic and circular nozzles.

The $\mathrm{AR}$ is $A R=1$ for the square nozzles and the circular nozzle. The mixing improves for square nozzles due to the introduction of smaller scales. Furthermore, as the blunt square and diamond-shaped are identical in shape, they introduce the same scales. Apparently, however, the orientation to the crossflow is also important with respect to mixing, such as blunt nozzle orientation improves mixing compared to pointed orientation.

The numerical accuracy of the computed mixing parameter is demonstrated in Figure 21 for circular nozzles as computed on three grids $(2.4,3.2$, and 3.8 million cells). In these cases, the scalar is set to zero at the wall. The sensitivity to the boundary condition is evaluated by computing the case with a zero-gradient condition for the scalar at the walls. The differences of the curves for varying cell sizes and wall boundary conditions (numerical and modeling parameters) are not as large as the differences for varying nozzles (a physical parameter). The numerical accuracy is therefore sufficient to demonstrate the effect of nozzle shape on the mixing parameter.

\subsection{Turbulence Statistics}

To give additional insight into the flow field, averages, rms, and turbulent scalar fluxes in the centerplane are discussed. Several features of the flow are identified to be potentially important for mixing, i.e. the trajectories, the strength of the CVP, the size of the fluctuations, and the distribution of energy among the various modes. The AR, introduction of smaller scales through non-circularity, and the bluntness of the nozzle are shown to have profound effects on these parameters. Figures 22 and 23 compare the average scalar profiles in the symmetry plane in the far-field at $z / D=8$. As noted before, such profiles have to be viewed with suspicion as they do not incorporate the three-dimensional effects discussed in Section 4.3. In the computed results, the elliptic nozzle with high AR has the highest peak, which is, however, a global peak as is demonstrated in Figure 16. The peaks of the curves for the circular nozzle and low AR elliptic nozzle are saddle points, i.e. maxima in y-direction but minima in x-direction (by symmetry) as shown in Figures 15 and 17 (for $z / D=12$ ). Compared to the experimental results, the magnitude of the scalar differs by $20-30 \%$ for the double-peaked distributions and by $40 \%$ for the singlepeaked distribution. This may be attributed to the presence of secondary flow features, to differences in boundary layer thickness, mass fluxes, and jet exit velocity profiles, and to the intrinsic uncertainty of LIF measurements. Taking the sensitivity of the flow field to physical parameters and uncertainties in the measurement technique into account, the agreement between the computation and experiment for the averages is as expected.

Figures 24 and 25 show the rms of the scalar at the same location. The curves are double-peaked due to large gradients on both windward and leeward side. The windward peak is slightly stronger than the leeward peak, consistent with the results of Su and Mungal [15]. The double-peaked rms profiles are evident in experiment and computation. The fluctuation in scalar is strongest for the nozzles which have the steepest scalar gradients in the mean. These are the nozzles with the global scalar peak on this line, i.e. the blunt square and the high AR elliptic nozzle. The discrepancy between experiment and computation is larger for the rms for which the computed and measured results differ by a factor of about two in the worst case. It is expected that comparisons of second moments will show larger discrepancies than for the first moments. Conceivable reasons for these differences between experiments and computations are discussed above.

Figures 26 and 27 reveal the transverse turbulent fluxes as obtained for the various nozzle shapes. Jet fluid is characterized by excess scalar concentration, excess $v$ momentum 


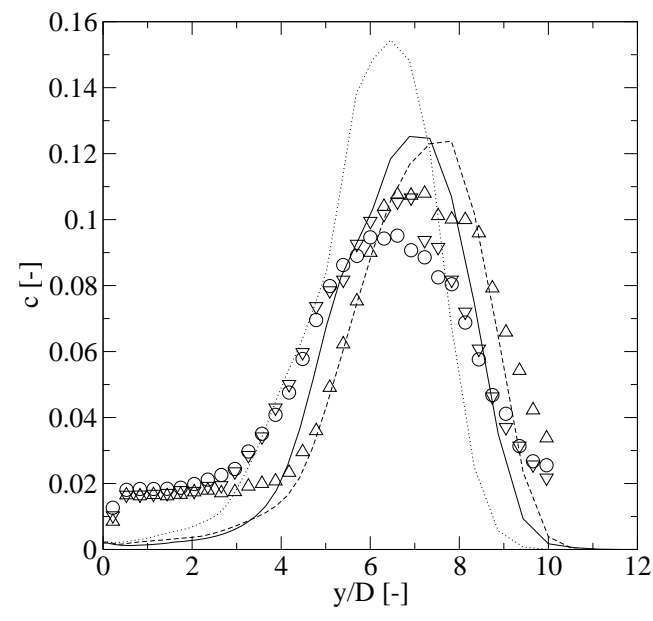

Figure 22: Computed (c) and measured (m) mean $\mathrm{c}$ for various nozzle shapes on a vertical line in the centerplane at $\mathrm{z} / \mathrm{D}=8$ : Round (m) $\bigcirc$, round $(\mathrm{c})-$, elliptic low AR $(\mathrm{m}) \triangle$, elliptic low AR (c) - - -, elliptic high AR (m) $\nabla$, elliptic high AR (c) ‥

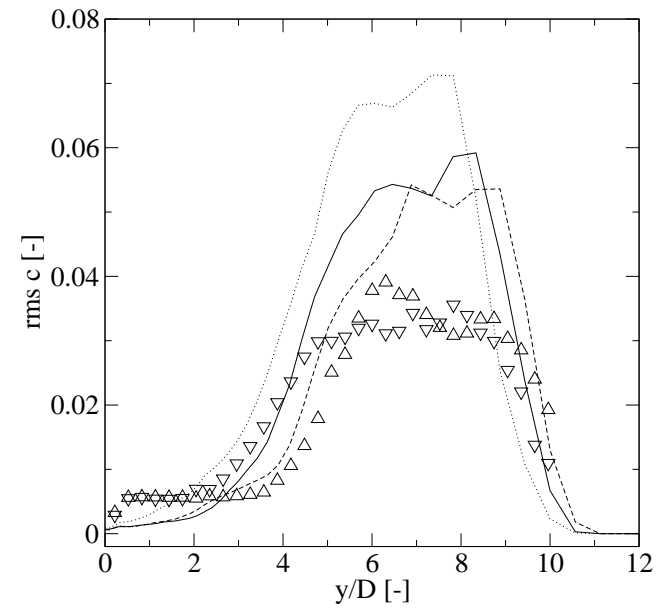

Figure 24: Computed (c) and measured (m) rms c for various nozzle shapes on a vertical line in the centerplane at $\mathrm{z} / \mathrm{D}=8$ : Round (c) -, elliptic low AR (m) $\triangle$, elliptic low AR (c) - - -, elliptic high AR (m) $\nabla$, elliptic high AR (c) ...

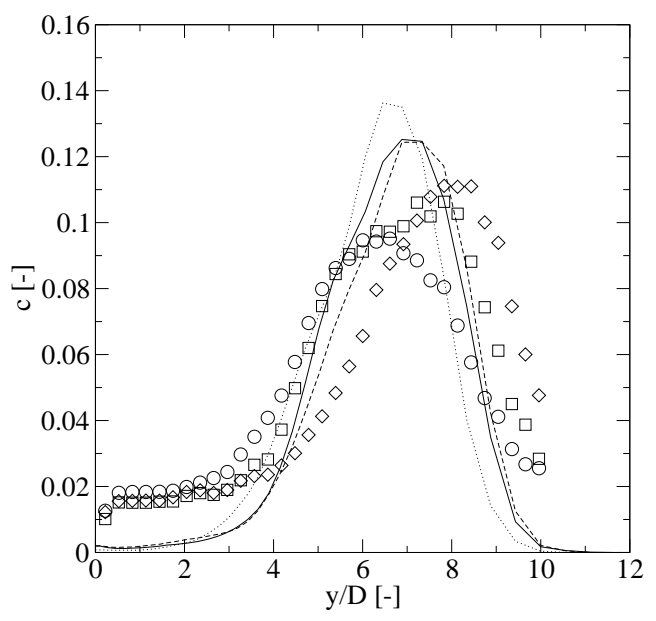

Figure 23: Computed (c) and measured (m) mean $\mathrm{c}$ for various nozzle shapes on a vertical line in the centerplane at $\mathrm{z} / \mathrm{D}=8$ : Round $(\mathrm{m})$ $\bigcirc$, round (c) - , square (m) $\square$, square (c) $\cdots$, diamond $(\mathrm{m}) \diamond$, diamond $(\mathrm{c})---$

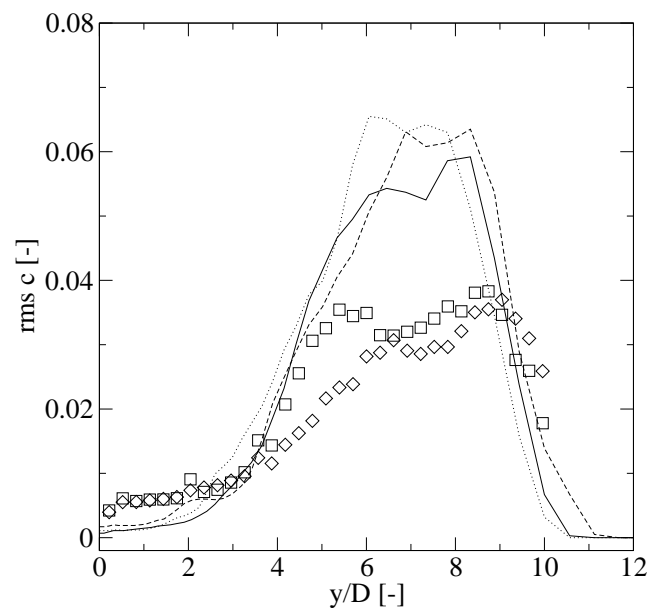

Figure 25: Computed (c) and measured (m) rms c for various nozzle shapes on a vertical line in the centerplane at $\mathrm{z} / \mathrm{D}=8$ : Round (c) -, square (m) $\square$, square (c) $\cdots$, diamond $(\mathrm{m}) \diamond$, diamond $(\mathrm{c})---$ 


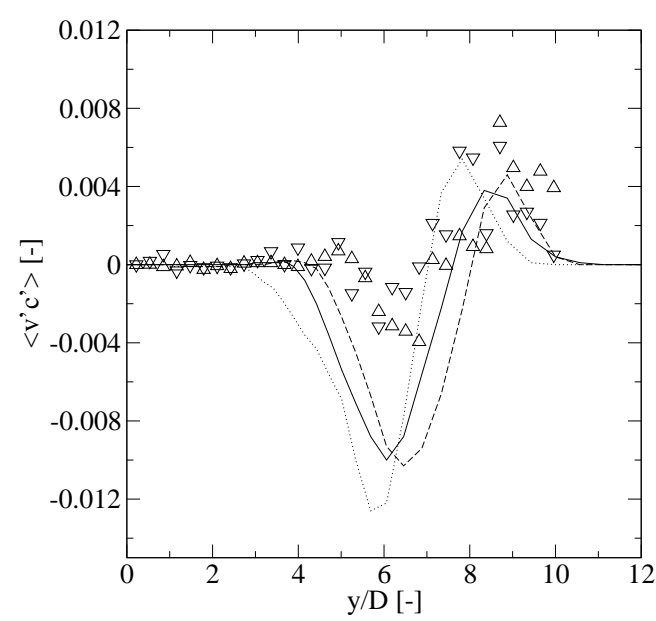

Figure 26: Computed (c) and measured (m) turbulent scalar flux $\left\langle v^{\prime} c^{\prime}\right\rangle$ on a vertical line in the centerplane at $\mathrm{z} / \mathrm{D}=8$ : Round (c) -, elliptic low AR (m) $\triangle$, elliptic low AR (c) --- , elliptic high $\mathrm{AR}(\mathrm{m}) \nabla$, elliptic high AR (c) ...

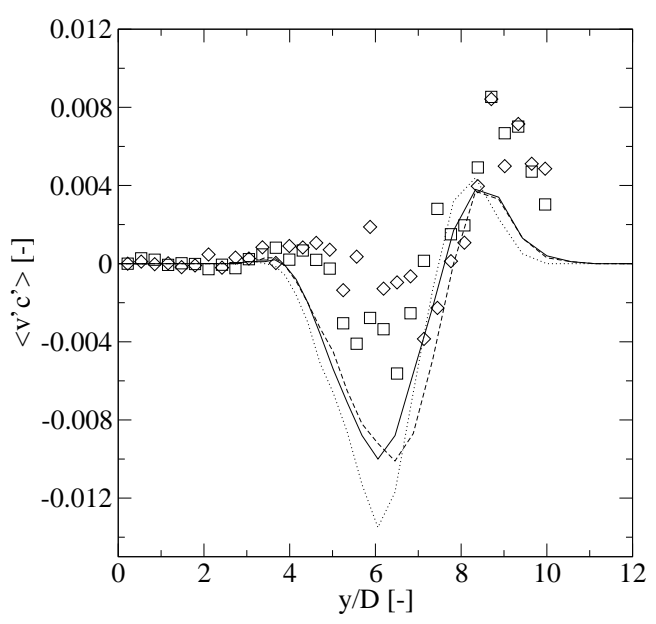

Figure 27: Computed (c) and measured (m) turbulent flux $\left\langle v^{\prime} c^{\prime}>\right.$ on a vertical line in the centerplane at $\mathrm{z} / \mathrm{D}=8$ : Round $(\mathrm{c})-$, square $(\mathrm{m}) \square$, square $(\mathrm{c}) \cdots$, diamond $(\mathrm{m})$ $\diamond$, diamond $(\mathrm{c})---$

flux, and deficit $w$ momentum flux. Crossflow fluid, on the other hand, is characterized by deficit scalar concentration, deficit $v$ momentum flux, and excess $w$ momentum flux. The shape of the scalar flux profiles are interpreted in terms of entrainment in $\mathrm{Su}$ and Mungal [15]. The turbulent scalar flux $\left\langle v^{\prime} c^{\prime}\right\rangle$ is negative below and positive above jet. Below the jet, excess upward (downward) velocities are associated with defect (excess) scalar concentration. Above the jet, excess downward (upward) velocities are correlated with defect (excess) scalar concentration. The turbulent fluxes in the symmetry plane are elevated for high AR elliptic and square nozzles. The profiles are qualitatively valid for the whole symmetry plane in the far-field, suggesting that these nozzle have stronger entrainment on the leeward side. The experimental data show qualitatively the same trends, but there is scatter in the data. These present computed profiles agree with the results of Su and Mungal [15]. There are, however, also some differences: Whereas Su and Mungal [15] find the windward cross-stream scalar flux to be largest, the present results show the cross-stream scalar flux in the wake of the jet to be larger than the windward flux by a factor of more than two.

The accuracy of the computed results are demonstrated for the turbulent flux $\left\langle v^{\prime} c^{\prime}\right\rangle$. The averages and the rms of the scalar are omitted for brevity. The difference in the solution for various grids is smaller than the difference in the solution for various nozzle geometries, and the numerical accuracy is therefore adequate. Furthermore, a case is computed which has a zero-gradient boundary condition at the walls for the scalar. The sensitivity to this change in boundary condition is as low as the sensitivity to the cell size.

\subsection{Counter-Gradient Transport}

In two-equation RANS turbulence models the turbulent scalar fluxes are unclosed in the governing equations and hence must be closed by modeling, most often by assuming an analogy between molecular diffusion and turbulent diffusion [30]:

$$
<v^{\prime} c^{\prime}>=-D_{T} \frac{\partial c}{\partial y}
$$




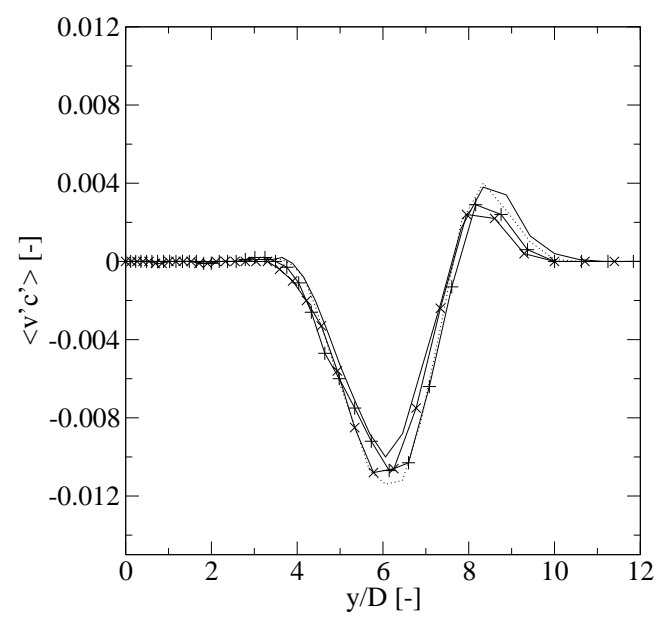

Figure 28: Numerical accuracy $\left\langle v^{\prime} c^{\prime}\right\rangle$, million cells: $--3.8,+-3.2, \mathrm{x}-2.4,---$ zero gradient boundary conditions

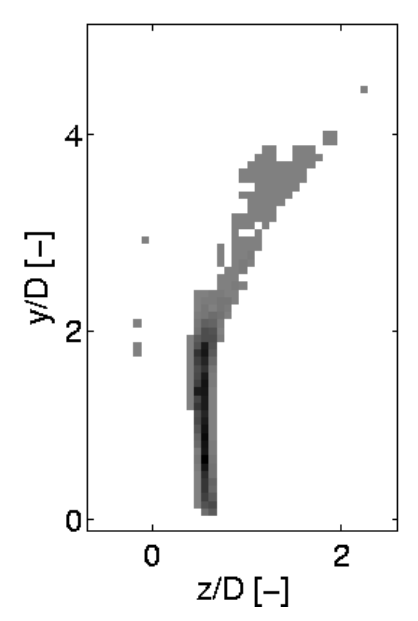

Figure 29: Measured regions of counter-gradient transport: $D_{T}<0$

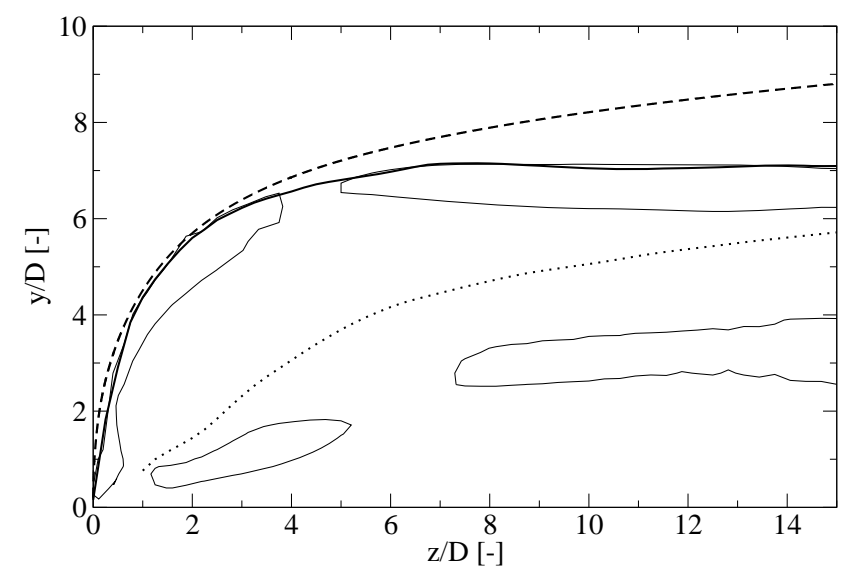

Figure 30: Computed regions of counter-gradient transport: $-D_{T}<0$, for orientation: Mean streamline --- , mean scalar trajectory (thick) -, mean vortex curve $\cdots$

According to the gradient diffusion hypothesis (Eq.10), the scalar must change signs where there is a peak in the average scalar. However, the peaks in average scalar (Figure 22) do not coincide with the location of sign change in the turbulent flux in $y$-direction (Figure 26), and therefore there must be large portions of counter-gradient transport in the flow field. A molecular diffusivity is normally positive, and so the analogy breaks down if the diffusivity coefficient $D_{T}$ attains a negative value. The regions with negative $D_{T}$ in the present flow field are plotted in Figure 29 and 30 for the measured and computed data, respectively. There is agreement between computation and experiments that there is a region of counter-gradient transport in the lee of the jet. For better orientation also the mean streamline, the trajectory based on the maximum scalar, and the mean vortex curve are shown in Fig.30. In non-isotropic turbulent flow, the turbulent scalar flux vector may not be aligned with the mean scalar gradient vector [30] and then the turbulent diffusivity (as defined in Eq. 10) can be negative. 


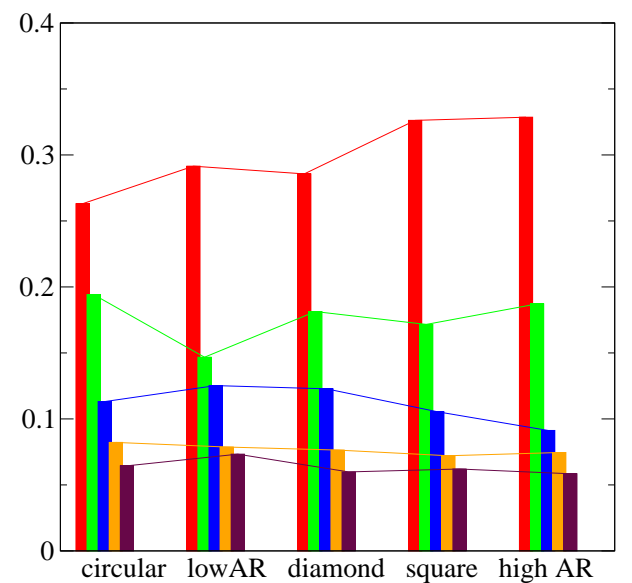

Figure 31: Normalized eigenvalues $\lambda_{N}$ of modes 1 to 5 for $\mathrm{u}$ velocity component

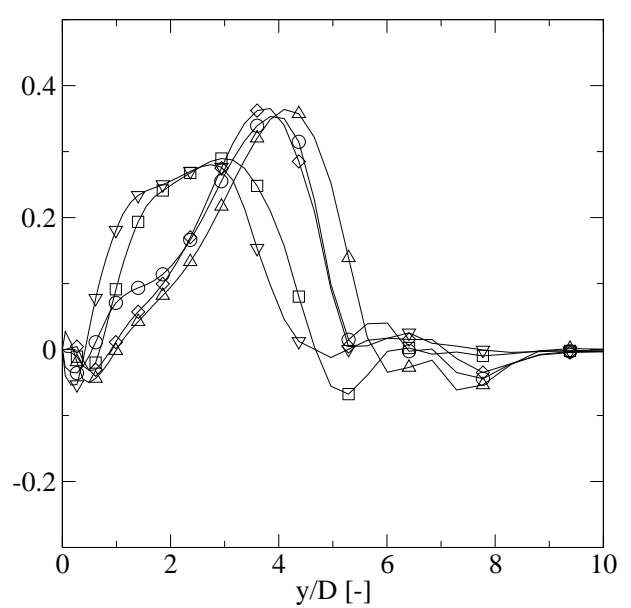

Figure 32: POD mode 1 for $\mathrm{u}$ velocity component, $\bigcirc$ - round, $\triangle$ - elliptic low AR, $\nabla$ elliptic high AR, $\square$ - square, $\diamond$ - diamond

\subsection{Proper Orthogonal Decomposition}

The qualitative differences in the distribution of the passive scalar in distal cross-sections must have a cause in the velocity field (see Figure 16 to Figure 19). The proper orthogonal decomposition (POD) of the lateral velocity further elucidates the eigenmodes in lateral direction. The proper orthogonal decomposition (POD) is a technique to extract spatially dominant features, i.e. coherent structures, from space-time data, in this case data obtained by LES. The data is decomposed into a set of $N$ empirical, mutually orthogonal eigenfunctions, or POD modes, $\phi_{n}(y)$, as follows in Eq. 11.

$$
u_{N}(y, t)=\sum_{n=1}^{N} a_{n}(t) \phi_{n}(y)
$$

It can be shown that it is a necessary condition that the POD modes satisfy the eigenvalue problem stated in Eq. 12 and Eq. 13 [31, 32]. The eigenvalue $\lambda_{n}$ is the variance of the random variable set in the direction of the eigenmode $\phi_{n}$. It has the dimension of the square of the random variable (see Eq. 13). In particular, if the random variable is a velocity, the eigenvalues have the dimension of specific energy. Therefore the eigenvalues are ordered by magnitude, and the set of the largest $N$ eigenvalues contains the most energetic modes $\phi_{n}$ : Coherent structures.

$$
\begin{aligned}
& A=<u u^{T}> \\
& A \phi_{n}=\lambda_{n} \phi_{n}
\end{aligned}
$$

Time-dependent data is sampled along a vertical line in the plane of symmetry at $\mathrm{z} / \mathrm{D}=6$. Figure 31 provides the POD eigenvalues of the lateral transverse modes. For each nozzle shape the largest six eigenvalues, which give the strength of transverse lateral modes, are plotted as bars. The lateral modes can be most clearly related to the occurrence of single- and double-peaked scalar distributions. Mode 1 for the blunt square and high AR elliptic nozzles have larger eigenvalues than they do for the other nozzles (Figure 31). Mode 1 describes coherent motion in transverse direction, and large eigenvalues imply stronger coherent transverse motion. The transverse lateral mode associated with the largest eigenvalue is plotted in Figure 32. First, there are striking differences in shape of 
mode 1 close to the wall. The blunt square and the high AR elliptic nozzles, which produce a single-peaked scalar distribution, have flat eigenmodes. Contrarily, the eigenmode of the circular, low AR elliptic, and diamond-shaped nozzles look slim and sharply peaked. In the former case the velocities in this mode are locked by correlation between $y / D \approx 1$ and $y / D \approx 4$, and in the latter case between $y / D \approx 2$ and $y / D \approx 5$. The sharp peak at $y / D \approx 4$ for the latter nozzles marks the position of their CVPs, which can be read from Figure 12 and Figure $13((z / D, y / D) \approx(6,4))$. Hence mode 1 for these nozzle locks lateral velocities in the CVP but does not give strong correlation to the direct lee of the jet below the CVP. For the square nozzle and the elliptic nozzle with high AR the velocities in the lee are also correlated to the velocities in the CVP.

\section{Conclusions}

A jet in crossflow is investigated using LES and simultaneous PIV/LIF. The global mixing performance is enhanced for nozzles which have a high aspect ratio, are blunt, and introduce smaller scales into the flow. This is related to the passive scalar distribution which can be single- or double peaked in cross-sectional planes, depending on nozzle shape and orientation. Proper orthogonal decomposition of the velocity fields reveals that this phenomenon may be related to the occurrence of large-scale coherent structures: Singlepeaked distributions occur in cases in which the first eigenmode has a large corresponding eigenvalue. The nozzles with single-peaked distribution, i.e. the elliptic nozzle with high aspect ratio and the blunt square nozzle, have superior mixing performance. Furthermore, it is shown that regions of counter-gradient transport are contained in the flow field.

\section{Acknowledgments}

This work was partially financed by the Swedish Energy Agency (STEM). The computational resources provided by LUNARC computing center at Lund University.

\section{References}

[1] Yuan, L., Street, R., and Ferziger, J., "Large-Eddy Simulation of a Round jet in Crossflow," J. Fluid Mech., Vol. 379, 1999, pp. 71-104.

[2] Gutmark, E. and Grinstein, F., "Flow Control with Noncircular Jets," Ann. Rev. Fluid Mech., Vol. 31, 1999, pp. 239-272.

[3] Yuan, L. and Street, R., "Trajectory and Entrainment of a Round Jet in Crossflow," Phys. Fluids, Vol. 10, No. 9, 1998, pp. 2323-2335.

[4] Ibrahim, I., Murugappan, S., and Gutmark, E., "Penetration, Mixing and Turbulent Structures of Circular and Non-Circular Jets in Cross Flow, AIAA-2005-0300," 43th AIAA Aerospace Science Meeting Proceedings, AIAA, 2005.

[5] Pratte, B. and Baines, W., "Profiles of the Round Turbulent Jet in a Cross Flow," J. Hydraulics Div., ASCE, Vol. 92, No. HY6, 1967, pp. 53-64.

[6] Kelso, R. and Smits, A., "Horseshoe Vortex Systems Resulting from the Interaction between a Laminar Boundary Layer and a Transverse Jet," Phys. Fluids, Vol. 7, No. 1, 1995, pp. 153-158. 
[7] Fric, T. and Roshko, A., "Vortical Structure in the Wake of a Tranverse Jet," J. Fluid Mech., Vol. 279, 1994, pp. 1-47.

[8] Joeng, J. and Hussain, F., "On the Identification of a Vortex," J. Fluid Mech., Vol. 285, 1995, pp. 69-94.

[9] Smith, S. and Mungal, M., "Mixing, Structure and Scaling of the Jet in Crossflow," J. Fluid Mech., Vol. 357, 1998, pp. 83-122.

[10] Kelso, R., Lim, T., and Perry, A., "An Experimental Study of Round Jets in CrossFlow," J. Fluid Mech., Vol. 306, 1996, pp. 111-144.

[11] Muppidi, S. and Mahesh, K., "Study of Trajectories of Jets in Crossflow Using Direct Numerical Simulations," J. Fluid Mech., Vol. 530, 2005, pp. 81-100.

[12] Kamotani, Y. and Greber, I., "Experiments on Confined Jets in Cross Flow," Tech. Rep. CR-2392, NASA, 1974.

[13] Haven, B. and Kurosaka, M., "Kidney and Anti-Kidney Vortices in Crossflow Jets," J. Fluid Mech., Vol. 352, 1997, pp. 27-64.

[14] Liscinsky, D., True, B., and Holdeman, J., "Crossflow Mixing of Noncircular Jets," J. Propulsion and Power, Vol. 12, No. 2, 1996, pp. 225-230.

[15] Su, L. and Mungal, M., "Simultaneous Measurements of Scalar and Velocity Field Evolution in Turbulent Crossflowing Jets," J. Fluid Mech., Vol. 513, 2004, pp. 1-45.

[16] Smagorinsky, J., "General Circulation Experiments with the Primitive Equations," Mon. Weather Rev., Vol. 91, No. 3, 1963, pp. 99-152.

[17] Bardina, J., Ferziger, J., and Reynolds, W., "Improved Subgrid Scale Models for Large Eddy Simulation, AIAA-80-1357," 13th Fluid and Plasma Dynamics Conference, AIAA, 1980.

[18] Germano, M., Piomelli, U., Moin, P., and Cabot, W., "A Dynamic Subgrid-Scale Eddy Viscosity Model," Phys. Fluids A, Vol. 3, 1991, pp. 1760-1765.

[19] Fureby, C. and Grinstein, F., "Large Eddy Simulation of High-Reynolds Free and Wall-Bounded Flows," J. Comp. Phys., Vol. 181, 2002, pp. 68-97.

[20] Kravchenko, A. and Moin, P., "On the Effect of Numerical Errors in Large Eddy Simulations of Turbulent Flows," J. Comp. Phys., Vol. 131, 1997, pp. 310-322.

[21] Ghosal, S., "An Analyis of Numerical Errors in Large-Eddy Simulations of Turbulence," J. Comp. Phys., Vol. 125, 1996, pp. 187-206.

[22] Olsson, M. and Fuchs, L., "Large eddy simulation of a forced semiconfined circular impinging jet," Phys. Fluids, Vol. 10, No. 2, 1998, pp. 476-486.

[23] Gullbrand, J. and Chow, F., "The effect of numerical errors and turbulence models in Large-Eddy Simulations of a channel flow, with and without explicit filtering," $J$. Fluid Mech., Vol. 495, 2003, pp. 323-341.

[24] Jiang, G.-S. and Shu, C.-W., "Efficient Implementation of Weighted ENO Schemes," J. Comp. Phys., Vol. 126, 1996, pp. 202-228. 
[25] Salewski, M., Stankovic, D., Fuchs, L., and Gutmark, E., "Coherent Structures in Circular and Non-Circular Jets in Crossflow, AIAA-2006-0907," 44th AIAA Aerospace Science Meeting Proceedings, AIAA, 2006.

[26] Fearn, R. and Weston, R., "Vorticity Associated with a Jet in a Cross Flow," AIAA J., Vol. 12, No. 10, 1974, pp. 1666-1671.

[27] Pope, S., "Ten Questions Concerning the Large-Eddy Simulation of Turbulent Flows," New J. Phys., Vol. 6, No. 35, 2004, pp. 1-24.

[28] Kamotani, Y. and Greber, I., "Experiments on a Turbulent Jet in a Cross Flow," AIAA J., Vol. 10, No. 11, 1972, pp. 1425-1429.

[29] Priére, C., Gicquel, L., Kaufmann, P., Krebs, W., and Poinsot, T., "Large Eddy Simulation Predictions of Mixing Enhancement for Jets in Cross-Flows," Journal of Turbulence, Vol. 5, No. 005, 2004, pp. 1-24.

[30] Pope, S., Turbulent Flows, Cambridge University Press, 2000.

[31] Holmes, P., Lumley, J., and Berkooz, B., Turbulence, Coherent Structures, Dynamical Systems and Symmetry, Cambridge University Press, 1996.

[32] Berkooz, G., Holmes, P., and Lumley, J., "The Proper Orthogonal Decomposition in the Analysis of Turbulent Flows," Ann. Rev. Fluid Mech., Vol. 25, 1993, pp. 539-575. 